\title{
Zero-one laws for connectivity in random key graphs
}

\author{
Osman Yağan and Armand M. Makowski, Fellow, IEEE
}

\begin{abstract}
The random key graph is a random graph naturally associated with the random key predistribution scheme introduced by Eschenauer and Gligor in the context of wireless sensor networks. For this class of random graphs we establish a new version of a conjectured zero-one law for graph connectivity as the number of nodes becomes unboundedly large. The results reported here complement and strengthen recent work on this conjecture by Blackburn and Gerke. In particular, the results are given under conditions which are more realistic for applications to wireless sensor networks.
\end{abstract}

Keywords: Wireless sensor networks, Key predistribution, Random key graphs, Graph connectivity, Zero-one laws.

\section{INTRODUCTION}

\section{A. Background}

Random key graphs, also known as uniform random intersection graphs, are random graphs that belong to the class of random intersection graphs [18]. They have appeared recently in application areas as diverse as clustering analysis [10], [11], collaborative filtering in recommender systems [14] and random key predistribution for wireless sensor networks (WSNs) [6], [7], [9].

For the sake of concreteness, we introduce this class of random graphs in this last context (hence the terminology). A WSN is a collection of spatially distributed sensors with limited capabilities for computations and wireless communications. It is envisioned that such networks will be used in applications such as battlefield surveillance, environment monitoring and traffic control, to name a few. In many settings, both military and civilian, network security will be a basic requirement for successful operations. However, traditional key exchange and distribution protocols are based on trusting third parties, and turn out to be inadequate for large-scale wireless sensor networks, e.g., see [9], [16], [20], [21] for

Manuscript received November 16, 2010; revised August 22, 2011. This work was supported by NSF Grant CCF-07290. The material in this paper was presented in part at the 2008 IEEE International Symposium on Information Theory (ISIT 2008), Toronto (Canada), June 2008, and at the 2009 IEEE International Symposium on Information Theory (ISIT 2009), Seoul (S Korea), June 2009.

O. Yağan was with the Department of Electrical and Computer Engineering, and the Institute for Systems Research, University of Maryland, College Park, MD 20742 USA. He is now with CyLab, Carnegie Mellon University, Pittsburgh, PA 15213 USA (e-mail: osmanyagan@gmail.com).

A. M. Makowski is with the Department of Electrical and Computer Engineering, and the Institute for Systems Research, University of Maryland, College Park, MD 20742 USA (e-mail: armand@isr.umd.edu).

Copyright (c) 2011 IEEE. Personal use of this material is permitted. However, permission to use this material for any other purposes must be obtained from the IEEE by sending a request to pubs-permissions@ieee.org. discussions of some of the challenges. To address some of the difficulties Eschenauer and Gligor [9] have recently proposed the following random key predistribution scheme:

Before deployment, each sensor in a WSN is independently assigned $K$ distinct cryptographic keys which are selected at random from a pool of $P$ keys (with $K<P$ ). These $K$ keys constitute the key ring of the node and are inserted into its memory. Two sensor nodes can then establish a secure link between them if they are within transmission range of each other and if their key rings have at least one key in common; see [9] for implementation details. A situation of particular interest is that of full visibility whereby nodes are all within communication range of each other. In that case a secure link can be established between two nodes if their key rings have at least one key in common. The resulting notion of adjacency defines the random key graph $\mathbb{K}(n ;(K, P))$ on the vertex set $\{1, \ldots, n\}$ where $n$ is the number of sensor nodes; see Section If for precise definitions.

A basic question concerning the scheme of Eschenauer and Gligor is its ability to achieve secure connectivity amongst participating nodes in the sense that a secure path exists between any pair of nodes. Therefore, under full visibility it is natural to seek conditions on $n, K$ and $P$ under which the random key graph $\mathbb{K}(n ;(K, P))$ constitutes a connected graph with high probability - The availability of such conditions would provide an encouraging indication of the feasibility of using this distribution scheme for WSNs. As discussed in Section IIII this search has lead to conjecturing the following zero-one law for graph connectivity in random key graphs: If the parameters $K$ and $P$ are scaled with $n$ according to

$$
\frac{K_{n}^{2}}{P_{n}}=\frac{\log n+\alpha_{n}}{n}, \quad n=1,2, \ldots
$$

for some sequence $\alpha: \mathbb{N}_{0} \rightarrow \mathbb{R}$, then it has been conjectured that

$$
\begin{gathered}
\lim _{n \rightarrow \infty} \mathbb{P}\left[\mathbb{K}\left(n ;\left(K_{n}, P_{n}\right)\right) \text { is connected }\right] \\
=\left\{\begin{array}{ccc}
0 & \text { if } \lim _{n \rightarrow \infty} \alpha_{n}=-\infty \\
1 & \text { if } \lim _{n \rightarrow \infty} \alpha_{n}=+\infty
\end{array}\right.
\end{gathered}
$$

This conjecture appeared independently in [1], [22]. The zero-one law (11)-(2) mimics a similar one for Erdős-Rényi graphs [2], and can be motivated from it by asymptotically matching the link assignment probabilities in these two classes of random graphs.

\section{B. Related work}

Recent results concerning the conjectured zero-one law (1)(2) are now surveyed: Di Pietro et al. have shown [7, Thm. 
4.6] that for large $n$, the random key graph will be connected with very high probability if $P_{n}$ and $K_{n}$ are selected such that

$$
K_{n} \geq 5, P_{n} \geq n \quad \text { and } \quad \frac{K_{n}^{2}}{P_{n}} \sim c \frac{\log n}{n}
$$

as soon as $c \geq 16$ They also observe that for large $n$, the random key graph will be disconnected with very high probability if the scaling satisfies

$$
\frac{K_{n}^{2}}{P_{n}}=o\left(\frac{\log n}{n}\right) .
$$

The zero-law in (2) has recently been established independently by Godehardt and Jaworski [10], Blackburn and Gerke [1], and Yağan and Makowski [22]. In all these papers, it was shown that

$$
\lim _{n \rightarrow \infty} \mathbb{P}\left[\mathbb{K}\left(n ;\left(K_{n}, P_{n}\right)\right) \text { contains no isolated nodes }\right]=0
$$

whenever $\lim _{n \rightarrow \infty} \alpha_{n}=-\infty$ in (11), a result which clearly implies the conjectured zero-law.

Blackburn and Gerke [1] also succeeded in generalizing the one-law result by Di Pietro et al. in a number of directions: Under the additional conditions

$$
K_{n} \geq 2 \quad \text { and } \quad P_{n} \geq n, \quad n=1,2, \ldots,
$$

they showed [1, Thm. 5] that

$$
\lim _{n \rightarrow \infty} \mathbb{P}\left[\mathbb{K}\left(n ;\left(K_{n}, P_{n}\right)\right) \text { is connected }\right]=1
$$

if

$$
\liminf _{n \rightarrow \infty} \frac{K_{n}^{2}}{P_{n}} \frac{n}{\log n}>1 .
$$

This result is weaker than the one-law in the conjecture (1)(2). However, in the process of establishing (4)-(5), they also show [1, Thm. 3] that the conjecture does hold in the special case $K_{n}=2$ for all $n=1,2, \ldots$ without any constraint on the size of the key pools, say $P_{n} \leq n$ or $n \leq P_{n}$. Specifically, the one-law in (2) is shown to hold whenever the scaling is done according to

$$
K_{n}=2, \quad \frac{4}{P_{n}}=\frac{\log n+\alpha_{n}}{n}, \quad n=1,2, \ldots
$$

as soon as $\lim _{n \rightarrow \infty} \alpha_{n}=\infty$. As pointed out by these authors, it is now easy to conclude that the one-law in (2) holds whenever $2 \leq K_{n} \leq P_{n}$ and $P_{n}=o\left(\frac{n}{\log n}\right)$; this corresponds to a constraint $P_{n} \ll n$.

\section{Contributions}

In this paper, we complement existing results concerning the conjecture (1)-(2) in several ways: We establish (Theorem 4.1) the one-law in (2) under the conditions $K_{n} \geq 2$ and $P_{n}=\Omega(n)$, i.e., $P_{n} \geq \sigma n$ for some $\sigma>0$. Since the zero-law in (2) has already been established [1], [10], [22], the validity of (11)-(2) thus follows whenever $P_{n}=\Omega(n)$ and $K_{n} \geq 2$.

This result already improves on the one-law (4)-(5) obtained by Blackburn and Gerke [1] under the condition (3). Moreover, as discussed earlier, these authors have established

\footnotetext{
${ }^{1}$ In the conference version of this work [6] Thm. 4.6] the result is claimed to hold for $c>8$.
}

the conjectured one-law in (2) under conditions very different from the ones used here, i..e., either $K_{n}=2$ or $K_{n} \geq 2$ with $P_{n}=o\left(\frac{n}{\log n}\right)$. In practical WSN scenarios it is expected that the size of the key pool will be much larger than the number of participating nodes [7], [9] and that key rings will contain more than two keys. In this context, our results concerning the full conjecture (1)-(2) are therefore given under more realistic conditions than earlier work.

The proof of the main result is lengthy and technically involved. However, in a parallel development, we have also shown in [26] that when $P_{n}=O\left(n^{\delta}\right)$ with $0<\delta<\frac{1}{2}$, the so-called small key pool case, elementary arguments can be used to establish a one-law for connectivity. This is an easy byproduct of the observation that connectivity is achieved in the random key graph whenever all possible key rings have been distributed to the participating nodes.

The results established in this paper were first announced in the conference paper [24] with an outline of the proofs; the full details were provided in an early draft [23] posted in January 2009. However, after completing this work, we learned of the independent work of Rybarczyk [17] concerning the conjecture (11)-(2) without any condition on the size of the key pool. Reference [17] deals mainly with the diameter and phase transition threshold of random key graphs, and uses branching process arguments similar to the ones given in [5]. The intermediary results, the so-called branching process lemmas, pave the way to a proof of the conjecture (11)-(2) by an approach very different from the one used here.

\section{The structure of the paper}

The paper is organized as follows: The class of random key graphs is formally introduced in Section [II A basis for the conjectured zero-one law is discussed in Section III and the main result of the paper, summarized as Theorem 4.1, is presented in Section IV A roadmap to the proof of Theorem 4.1 is given in Section $\nabla$ The approach is similar to the one used for proving the one-law for graph connectivity in ErdősRényi graphs [2, p. 164], [8, Section 3.4, p. 40] , [19, p. 304]; see (9)- 10). Here as well, we focus on the probability that the random key graph is not connected and yet has no isolated nodes. We then seek to show that this probability becomes vanishingly small as $n$ grows large under the appropriate scaling. As in the classical case this is achieved through a combination of judicious bounding arguments, the starting point being the well-known bound (43) on the probability of interest. However, in order for these arguments to successfully go through, we found it necessary to restrict attention to a subclass of structured scalings (referred throughout as strongly admissible scalings). In Section VI a reduction argument shows that we need only establish the desired one-law for such strongly admissible scalings. The explanation of the right handside of (1) as a proxy for link assignment in the limiting regime is revealed through a useful equivalence developed in Section VII

With these technical prerequisites in place, the needed bounding arguments are then developed in Section VIII, Section IX and Section $\mathrm{X}$ and the final steps of the proof of Theorem 4.1 are outlined in Section XI. The final sections 
of the paper, namely Section XII through Section XVII are devoted to the various technical steps needed to complete the arguments outlined in Section XI

\section{E. Notation and conventions}

A word on the notation and conventions in use: All limiting statements, including asymptotic equivalences, are understood with $n$ going to infinity. The random variables (rvs) under consideration are all defined on the same probability triple $(\Omega, \mathcal{F}, \mathbb{P})$. Probabilistic statements are made with respect to this probability measure $\mathbb{P}$, and we denote the corresponding expectation operator by $\mathbb{E}$. The indicator function of an event $E$ is denoted by $\mathbf{1}[E]$. For any discrete set $S$ we write $|S|$ for its cardinality.

\section{RANDOM KEY GRAPHS}

Random key graphs are parametrized by the number $n$ of nodes, the size $P$ of the key pool and the size $K$ of each key ring with $K \leq P$. To lighten the notation we often group the integers $P$ and $K$ into the ordered pair $\theta \equiv(K, P)$.

Nodes are labelled $i, \ldots, n$ while keys are labelled $1, \ldots, P$. For each node $i=1, \ldots, n$, let $K_{i}(\theta)$ denote the random set of $K$ distinct keys assigned to node $i$. We can think of $K_{i}(\theta)$ as an $\mathcal{P}_{K}$-valued rv where $\mathcal{P}_{K}$ denotes the collection of all subsets of $\{1, \ldots, P\}$ which contain exactly $K$ elements Obviously, we have $\left|\mathcal{P}_{K}\right|=\left(\begin{array}{c}P \\ K\end{array}\right)$. The rvs $K_{1}(\theta), \ldots, K_{n}(\theta)$ are assumed to be i.i.d. rvs, each of which is uniformly distributed over $\mathcal{P}_{K}$ with

$$
\mathbb{P}\left[K_{i}(\theta)=S\right]=\left(\begin{array}{l}
P \\
K
\end{array}\right)^{-1}, \quad S \in \mathcal{P}_{K}
$$

for all $i=1, \ldots, n$. This corresponds to selecting keys randomly and without replacement from the key pool.

Distinct nodes $i, j=1, \ldots, n$ are said to be adjacent if they share at least one key in their key rings, namely

$$
K_{i}(\theta) \cap K_{j}(\theta) \neq \emptyset,
$$

in which case an undirected link is assigned between nodes $i$ and $j$. The resulting random graph defines the random key graph on the vertex set $\{1, \ldots, n\}$, hereafter denoted by $\mathbb{K}(n ; \theta)$. For distinct $i, j=1, \ldots, n$, it is a simple matter to check that

$$
\mathbb{P}\left[K_{i}(\theta) \cap K_{j}(\theta)=\emptyset\right]=q(\theta)
$$

with

$$
q(\theta)= \begin{cases}0 & \text { if } P<2 K \\
\frac{\left(\begin{array}{l}
P-K \\
K
\end{array}\right)}{\left(\begin{array}{l}
P \\
K
\end{array}\right)} & \text { if } 2 K \leq P,\end{cases}
$$

whence the probability of edge occurrence between any two nodes is equal to $1-q(\theta)$. The expression (6) and others given later are simple consequences of the often used fact that

$$
\mathbb{P}\left[S \cap K_{i}(\theta)=\emptyset\right]= \begin{cases}0 & \text { if }|S|>P-K \\
\frac{\left(\begin{array}{l}
P-|S| \\
K
\end{array}\right)}{\left(\begin{array}{l}
P \\
K
\end{array}\right)} & \text { if }|S| \leq P-K\end{cases}
$$

with $S$ a subset of $\{1, \ldots, P\}$. The case $P<2 K$ corresponds to an edge existing between every pair of nodes, so that
$\mathbb{K}(n ; \theta)$ coincides with the complete graph on the vertex set $\{1, \ldots, n\}$. Also, we always have $0 \leq q(\theta)<1$ with $q(\theta)>0$ if and only if $2 K \leq P$.

Random key graphs form a subclass in the family of random intersection graphs. However, the model adopted here differs from the random intersection graphs discussed by SingerCohen et al. in [13], [18] where each node is assigned a key ring, one key at a time according to a Bernoulli-like mechanism (so that each key ring has a random size and has positive probability of being empty). Both subclasses are subsumed by the more general random intersection graph model discussed by Godehardt et al. [10], [11].

Throughout, with $n=2,3, \ldots$, and positive integers $K$ and $P$ such that $K \leq P$, let $P(n ; \theta)$ denote the probability that the random key graph $\mathbb{K}(n ; \theta)$ is connected, namely

$$
P(n ; \theta):=\mathbb{P}[\mathbb{K}(n ; \theta) \text { is connected }], \quad \theta=(K, P) .
$$

\section{A BASIS FOR THE CONJECTURE}

As indicated earlier, we wish to select $P$ and $K$ so that $P(n ; \theta)$ is as large (i.e., as close to one) as possible. We outline below a possible approach which is inspired by the discussion on this issue given by Eschenauer and Gligor in their original work [9]; see also the discussion in [6], [7],

(i) Let $\mathbb{G}(n ; p)$ denote the Erdős-Rényi graph on $n$ vertices with edge probability $p(0<p \leq 1)$ [2], [8], [12]. Despite strong similarities, the random graph $\mathbb{K}(n ; \theta)$ is not an ErdôsRényi graph $\mathbb{G}(n ; p)$. This is so because edge assignments are independent in $\mathbb{G}(n ; p)$ but can be correlated in $\mathbb{K}(n ; \theta)$. Yet, setting aside this (inconvenient) fact, we note that $\mathbb{K}(n ; \theta)$ can be matched naturally to an Erdős-Rényi graph $\mathbb{G}(n ; p)$ with $p$ and $\theta$ related through

$$
p=1-q(\theta)
$$

This constraint ensures that link assignment probabilities in $\mathbb{K}(n ; \theta)$ and $\mathbb{G}(n ; p)$ coincide. Moreover, under $(8)$ it is easy to check that the degree of a node in either random graph is a Binomial rv with the same parameters, namely $n-1$ and $p=1-q(\theta) \sqrt{2}$ Given that the degree distributions in a random graph are often taken (perhaps mistakenly) as a good indicator of its connectivity properties, it is tempting to conclude that the zero-one law for graph connectivity in random key graphs can be inferred from the analog result for Erdős-Rényi graphs when matched through the condition (8).

(ii) To perform such a "transfer," we first recall that in Erdős-Rényi graphs the property of graph connectivity is known to exhibit the following zero-one law [2]: If we scale the edge assignment probability $p$ according to

$$
p_{n}=\frac{\log n+\alpha_{n}}{n}, \quad n=1,2, \ldots
$$

for some sequence $\alpha: \mathbb{N}_{0} \rightarrow \mathbb{R}$, then

$$
\begin{aligned}
\lim _{n \rightarrow \infty} \mathbb{P}\left[\mathbb{G}\left(n ; p_{n}\right) \text { is connected }\right] \\
=\left\{\begin{array}{lll}
0 & \text { if } \lim _{n \rightarrow \infty} \alpha_{n}=-\infty \\
1 & \text { if } \lim _{n \rightarrow \infty} \alpha_{n}=+\infty
\end{array}\right.
\end{aligned}
$$

${ }^{2}$ For Erdős-Rényi graphs this result is well known, while for random key graphs this characterization is a straightforward consequence of 7. 
(iii) Under the matching condition (8), these classical results suggest scaling the parameters $K$ and $P$ with $n$ according to

$$
1-\frac{\left(\begin{array}{c}
P_{n}-K_{n} \\
K_{n}
\end{array}\right)}{\left(\begin{array}{l}
P_{n} \\
K_{n}
\end{array}\right)}=\frac{\log n+\alpha_{n}}{n}, \quad n=1,2, \ldots
$$

for some sequence $\alpha: \mathbb{N}_{0} \rightarrow \mathbb{R}$. In view of (10) it is then not too unreasonable to expect that the zero-one law

$$
\lim _{n \rightarrow \infty} P\left(n ; \theta_{n}\right)= \begin{cases}0 & \text { if } \lim _{n \rightarrow \infty} \alpha_{n}=-\infty \\ 1 & \text { if } \lim _{n \rightarrow \infty} \alpha_{n}=+\infty\end{cases}
$$

should hold (possibly under some additional assumptions).

Of course, for this approach to be operationally useful, a good approximation to the right handside of 8 is needed. Eschenauer and Gligor provided such an approximation with the help of Stirling's formula. However, as already indicated by Di Pietro et al. [6], [7], it is easy to check that

$$
1-\frac{\left(\begin{array}{c}
P-K \\
K
\end{array}\right)}{\left(\begin{array}{l}
P \\
K
\end{array}\right)} \simeq \frac{K^{2}}{P}
$$

under natural assumptions; see Lemma 7.3. Thus, if instead of scaling the parameters according to 11 , we scale them according to

$$
\frac{K_{n}^{2}}{P_{n}}=\frac{\log n+\alpha_{n}}{n}, \quad n=1,2, \ldots
$$

then it is natural to conjecture that the zero-one law (12) should still hold.

While this transfer technique could in principle be applied to other graph properties, it may not always yield the correct form for the zero-one law; see the papers [25], [27] for results concerning the existence of triangles in random key graphs.

\section{THE MAIN RESULT}

Any pair of functions $P, K: \mathbb{N}_{0} \rightarrow \mathbb{N}_{0}$ defines a scaling provided the natural conditions

$$
K_{n} \leq P_{n}, \quad n=1,2, \ldots
$$

are satisfied. We can always associate with it a sequence $\alpha$ : $\mathbb{N}_{0} \rightarrow \mathbb{R}$ through the relation

$$
\frac{K_{n}^{2}}{P_{n}}=\frac{\log n+\alpha_{n}}{n}, \quad n=1,2, \ldots
$$

Just set

$$
\alpha_{n}:=n \frac{K_{n}^{2}}{P_{n}}-\log n, \quad n=1,2, \ldots
$$

We refer to this sequence $\alpha: \mathbb{N}_{0} \rightarrow \mathbb{R}$ as the deviation function associated with the scaling $P, K: \mathbb{N}_{0} \rightarrow \mathbb{N}_{0}$. As the terminology suggests, the deviation function measures by how much the scaling deviates from the critical scaling $\frac{\log n}{n}$.

A scaling $P, K: \mathbb{N}_{0} \rightarrow \mathbb{N}_{0}$ is said to be admissible if

$$
2 \leq K_{n}
$$

for all $n=1,2, \ldots$ sufficiently large. The main result of this paper can now be stated as follows.
Theorem 4.1: Consider an admissible scaling $P, K: \mathbb{N}_{0} \rightarrow$ $\mathbb{N}_{0}$ with deviation function $\alpha: \mathbb{N}_{0} \rightarrow \mathbb{R}$ determined through (14). We have

$$
\lim _{n \rightarrow \infty} P\left(n ; \theta_{n}\right)=0 \quad \text { if } \lim _{n \rightarrow \infty} \alpha_{n}=-\infty .
$$

On the other hand, if there exists some $\sigma>0$ such that

$$
\sigma n \leq P_{n}
$$

for all $n=1,2, \ldots$ sufficiently large, then we have

$$
\lim _{n \rightarrow \infty} P\left(n ; \theta_{n}\right)=1 \text { if } \lim _{n \rightarrow \infty} \alpha_{n}=\infty .
$$

The condition (16) is sometimes expressed as $P_{n}=\Omega(n)$ and is slightly weaker than the growth condition at (3) used by Blackburn and Gerke [1]. Furthermore, Theorem 4.1 implies the much weaker one-law (4)-(5). We also note that the onelaw in Theorem 4.1 cannot hold if the condition (15) fails. This is a simple consequence of the following observation; see [28] for details.

Lemma 4.2: For any mapping $P: \mathbb{N}_{0} \rightarrow \mathbb{N}_{0}$ for which the limit $\lim _{n \rightarrow \infty} P_{n}$ exists (possibly infinite), we have

$$
\lim _{n \rightarrow \infty} P\left(n ;\left(1, P_{n}\right)\right)= \begin{cases}0 & \text { if } \lim _{n \rightarrow \infty} P_{n}>1 \\ 1 & \text { if } \lim _{n \rightarrow \infty} P_{n}=1 .\end{cases}
$$

\section{A ROADMAP FOR THE PROOF OF THEOREM 4.1}

Fix $n=2,3, \ldots$ and consider positive integers $K$ and $P$ such that $2 \leq K \leq P$. We define the events

$$
C_{n}(\theta):=[\mathbb{K}(n ; \theta) \text { is connected }]
$$

and

$$
I_{n}(\theta):=[\mathbb{K}(n ; \theta) \text { contains no isolated nodes }] .
$$

If the random key graph $\mathbb{K}(n ; \theta)$ is connected, then it does not contain isolated nodes, whence $C_{n}(\theta)$ is a subset of $I_{n}(\theta)$, and the conclusions

$$
\mathbb{P}\left[C_{n}(\theta)\right] \leq \mathbb{P}\left[I_{n}(\theta)\right]
$$

and

$$
\mathbb{P}\left[C_{n}(\theta)^{c}\right]=\mathbb{P}\left[C_{n}(\theta)^{c} \cap I_{n}(\theta)\right]+\mathbb{P}\left[I_{n}(\theta)^{c}\right]
$$

obtain.

In [22], we established the following zero-one law for the absence of isolated nodes by the method of first and second moments applied to the number of isolated nodes.

Theorem 5.1: For any admissible scaling $P, K: \mathbb{N}_{0} \rightarrow \mathbb{N}_{0}$, it holds that

$$
\lim _{n \rightarrow \infty} \mathbb{P}\left[I_{n}\left(\theta_{n}\right)\right]=\left\{\begin{array}{cc}
0 & \text { if } \lim _{n \rightarrow \infty} \alpha_{n}=-\infty \\
1 & \text { if } \lim _{n \rightarrow \infty} \alpha_{n}=+\infty
\end{array}\right.
$$

where the deviation function $\alpha: \mathbb{N}_{0} \rightarrow \mathbb{R}$ is determined through (14).

This result was also obtained independently by Blackburn and Gerke [1] and Godehardt and Jaworski [10]. In this last paper the authors show the stronger result that the number 
of isolated nodes is asymptotically Poisson distributed with parameter $e^{-c}$ under scalings of the form (14) with deviation function satisfying $\lim _{n \rightarrow \infty} \alpha_{n}=c$ for some finite scalar $c$.

Taken together with Theorem 5.1 the relations (18) and (19) pave the way to proving Theorem 4.1. Indeed, pick an admissible scaling $P, K: \mathbb{N}_{0} \rightarrow \mathbb{N}_{0}$ with deviation function $\alpha: \mathbb{N}_{0} \rightarrow \mathbb{R}$. If $\lim _{n \rightarrow \infty} \alpha_{n}=-\infty$, then $\lim _{n \rightarrow \infty} \mathbb{P}\left[I_{n}\left(\theta_{n}\right)\right]=0$ by the zero-law for the absence of isolated nodes, whence $\lim _{n \rightarrow \infty} \mathbb{P}\left[C_{n}\left(\theta_{n}\right)\right]=0$ with the help of (18). If $\lim _{n \rightarrow \infty} \alpha_{n}=\infty$, then $\lim _{n \rightarrow \infty} \mathbb{P}\left[I_{n}\left(\theta_{n}\right)\right]=1$ by the one-law for the absence of isolated nodes, and the desired conclusion $\lim _{n \rightarrow \infty} \mathbb{P}\left[C_{n}\left(\theta_{n}\right)\right]=1$ (or equivalently, $\lim _{n \rightarrow \infty} \mathbb{P}\left[C_{n}\left(\theta_{n}\right)^{c}\right]=0$ ) will follow via (19] if we show that

$$
\lim _{n \rightarrow \infty} \mathbb{P}\left[C_{n}\left(\theta_{n}\right)^{c} \cap I_{n}\left(\theta_{n}\right)\right]=0 .
$$

We shall do this by finding a sufficiently tight upper bound on the probability in 20 and then showing that it goes to zero as well. While the additional condition (16) plays a crucial role in carrying out this argument, a number of additional assumptions will be imposed on the admissible scaling under consideration. This is done mostly for technical reasons in that it leads to simpler proofs. Eventually these additional conditions will be removed to ensure the desired final result, namely 17 under (16), e.g., see Section VI for details.

With this in mind, the admissible scaling $P, K: \mathbb{N}_{0} \rightarrow \mathbb{N}_{0}$ is said to be strongly admissible if its deviation function $\alpha$ : $\mathbb{N}_{0} \rightarrow \mathbb{R}$ satisfies the additional growth condition

$$
\alpha_{n}=o(n) \text {. }
$$

Strong admissibility has the following useful implications: Under (21) it is always the case from (14) that

$$
\lim _{n \rightarrow \infty} \frac{K_{n}^{2}}{P_{n}}=0 .
$$

Since $1 \leq K_{n} \leq K_{n}^{2}$ for all $n=1,2, \ldots$, this last convergence implies

$$
\lim _{n \rightarrow \infty} \frac{K_{n}}{P_{n}}=0 \text { and } \quad \lim _{n \rightarrow \infty} P_{n}=\infty .
$$

As a result, we have

$$
2 K_{n} \leq P_{n}
$$

for all $n=1,2, \ldots$ sufficiently large, and the random key graph does not degenerate into a complete graph under a strongly admissible scaling. Finally, in Lemma 7.3 we show that (22) suffices to imply

$$
1-q\left(\theta_{n}\right) \sim \frac{K_{n}^{2}}{P_{n}}
$$

This is discussed in Section VII and provides the appropriate version of 13.

\section{A REDUCTION STEP}

The relevance of the notion of strong admissibility flows from the following fact.

Lemma 6.1: Consider an admissible scaling $K, P: \mathbb{N}_{0} \rightarrow$ $\mathbb{N}_{0}$ whose deviation sequence $\alpha: \mathbb{N}_{0} \rightarrow \mathbb{R}$ satisfies

$$
\lim _{n \rightarrow \infty} \alpha_{n}=\infty
$$

Assume there exists some $\sigma>0$ such that (16) holds for all $n=1,2, \ldots$ sufficiently large. Then, there always exists an admissible scaling $\tilde{K}, \tilde{P}: \mathbb{N}_{0} \rightarrow \mathbb{N}_{0}$ with

$$
\tilde{K}_{n} \leq K_{n} \quad \text { and } \quad \tilde{P}_{n}=P_{n}, \quad n=1,2, \ldots
$$

whose deviation function $\tilde{\alpha}: \mathbb{N}_{0} \rightarrow \mathbb{R}$ satisfies both conditions

$$
\lim _{n \rightarrow \infty} \tilde{\alpha}_{n}=\infty \quad \text { and } \quad \tilde{\alpha}_{n}=o(n) .
$$

Proof. For each $n=1,2, \ldots$, set

$$
K_{n}^{\star}:=\sqrt{P_{n} \cdot \frac{\log n+\alpha_{n}^{\star}}{n}} \text { where } \alpha_{n}^{\star}:=\min \left(\alpha_{n}, \log n\right) .
$$

The properties

$$
\lim _{n \rightarrow \infty} \alpha_{n}^{\star}=\infty
$$

and

$$
\alpha_{n}^{\star}=o(n)
$$

are immediate by construction.

Now define the scaling $\tilde{K}, \tilde{P}: \mathbb{N}_{0} \rightarrow \mathbb{N}_{0}$ by

$$
\tilde{K}_{n}:=\left\lceil K_{n}^{\star}\right\rceil, \tilde{P}_{n}=P_{n}, \quad n=1,2, \ldots
$$

We get $K_{n}^{\star} \leq K_{n}$ for all $n=1,2, \ldots$ since $\alpha_{n}^{\star} \leq \alpha_{n}$, whence $\tilde{K}_{n} \leq K_{n}$ by virtue of the fact that $K_{n}$ is always an integer. This establishes (26).

Next, observe that $\tilde{K}_{n}=1$ if and only $K_{n}^{\star} \leq 1$, a condition which occurs only when

$$
P_{n}\left(\log n+\alpha_{n}^{\star}\right) \leq n .
$$

This last inequality can only hold for a finite number of values of $n$. Otherwise, there would exist a countably infinite subset $N$ of $\mathbb{N}_{0}$ such that both (16) and (30) simultaneously hold on $N$. In that case, we conclude that

$$
\sigma\left(\log n+\alpha_{n}^{\star}\right) \leq 1, \quad n \in N
$$

and this is a clear impossibility in view of (28). Together with (26) this establishes the admissibility of the scaling $\tilde{K}, \tilde{P}$ : $\mathbb{N}_{0} \rightarrow \mathbb{N}_{0}$.

Fix $n=1,2, \ldots$. The definitions imply $K_{n}^{\star} \leq \tilde{K}_{n}<1+$ $K_{n}^{\star}$, and upon squaring we get the inequalities

$$
P_{n} \cdot \frac{\log n+\alpha_{n}^{\star}}{n} \leq \tilde{K}_{n}^{2}
$$

and

$$
\tilde{K}_{n}^{2}<1+2 \sqrt{P_{n} \cdot \frac{\log n+\alpha_{n}^{\star}}{n}}+P_{n} \cdot \frac{\log n+\alpha_{n}^{\star}}{n} .
$$

The deviation sequence $\tilde{\alpha}: \mathbb{N}_{0} \rightarrow \mathbb{R}$ of the newly defined scaling (26) is determined through

$$
\frac{\tilde{K}_{n}^{2}}{\tilde{P}_{n}}=\frac{\log n+\tilde{\alpha}_{n}}{n}, \quad n=1,2, \ldots
$$

Using the two inequalities above we then conclude that

$$
\alpha_{n}^{\star} \leq \tilde{\alpha}_{n}
$$

and

$$
\frac{\tilde{\alpha}_{n}}{n}<\frac{1}{P_{n}}+2 \sqrt{\frac{1}{P_{n}} \cdot \frac{\log n+\alpha_{n}^{\star}}{n}}+\frac{\alpha_{n}^{\star}}{n} .
$$


It is now plain from (28) and (31) that the first half of 27 holds. Next, by combining (31) and (32) we get

$$
\frac{\alpha_{n}^{\star}}{n} \leq \frac{\tilde{\alpha}_{n}}{n}<\frac{1}{P_{n}}+2 \sqrt{\frac{1}{P_{n}} \cdot \frac{\log n+\alpha_{n}^{\star}}{n}}+\frac{\alpha_{n}^{\star}}{n} .
$$

Letting $n$ go to infinity in (33) and using (29) we conclude to the second half of (27) since $\lim _{n \rightarrow \infty} P_{n}=\infty$ by virtue of (16).

The scaling $\tilde{K}, \tilde{P}: \mathbb{N}_{0} \rightarrow \mathbb{N}_{0}$ defined at (26) is strongly admissible and still satisfies the condition (16), and an easy coupling argument based on (26) shows that

$$
P\left(n ; \tilde{\theta}_{n}\right) \leq P\left(n ; \theta_{n}\right), \quad n=2,3, \ldots
$$

Therefore, we need only show (17) under (16) for strongly admissible scalings. As a result, in view of the discussion leading to 20) it suffices to establish the following result, to which the remainder of the paper is devoted.

Proposition 6.2: Consider any strongly admissible scaling $P, K: \mathbb{N}_{0} \rightarrow \mathbb{N}_{0}$ whose deviation function $\alpha: \mathbb{N}_{0} \rightarrow \mathbb{R}$ satisfies $\lim _{n \rightarrow \infty} \alpha_{n}=\infty$. Under the condition (16), we have

$$
\lim _{n \rightarrow \infty} \mathbb{P}\left[C_{n}\left(\theta_{n}\right)^{c} \cap I_{n}\left(\theta_{n}\right)\right]=0 .
$$

Proposition 6.2 shows that in random key graphs, graph connectivity is asymptotically equivalent to the absence of isolated nodes under any strongly admissible scaling whose deviation function $\alpha: \mathbb{N}_{0} \rightarrow \mathbb{R}$ satisfies $\lim _{n \rightarrow \infty} \alpha_{n}=\infty$ under the condition (16).

\section{The EQuivalence 25}

To establish the key equivalence (25) we start with simple bounds which prove useful in a number of places. Full details are available in [23], [28].

Lemma 7.1: For positive integers $K, L$ and $P$ such that $K+$ $L \leq P$, we have

$$
\left(1-\frac{L}{P-K}\right)^{K} \leq \frac{\left(\begin{array}{c}
P-L \\
K
\end{array}\right)}{\left(\begin{array}{l}
P \\
K
\end{array}\right)} \leq\left(1-\frac{L}{P}\right)^{K}
$$

whence

$$
\frac{\left(\begin{array}{c}
P-L \\
K
\end{array}\right)}{\left(\begin{array}{l}
P \\
K
\end{array}\right)} \leq e^{-K \cdot \frac{L}{P}}
$$

Applying Lemma 7.1 (with $L=K$ ) to the expression (6) yields the following bounds.

Lemma 7.2: With positive integers $K$ and $P$ such that $2 K \leq$ $P$, we have

$$
1-e^{-\frac{K^{2}}{P}} \leq 1-q(\theta) \leq \frac{K^{2}}{P-K} .
$$

A little bit more than 25) can then be said.

Lemma 7.3: For any scaling $P, K: \mathbb{N}_{0} \rightarrow \mathbb{N}_{0}$, it holds that

$$
\lim _{n \rightarrow \infty} q\left(\theta_{n}\right)=1
$$

if and only if

$$
\lim _{n \rightarrow \infty} \frac{K_{n}^{2}}{P_{n}}=0
$$

and under either condition we have the asymptotic equivalence

$$
1-q\left(\theta_{n}\right) \sim \frac{K_{n}^{2}}{P_{n}}
$$

On several occasions, we will rely on (38) through the following equivalent formulation: For every $\delta$ in $(0,1)$ there exists a finite integer $n^{\star}(\delta)$ such that

$$
(1-\delta) \frac{K_{n}^{2}}{P_{n}} \leq 1-q\left(\theta_{n}\right) \leq(1+\delta) \frac{K_{n}^{2}}{P_{n}}
$$

whenever $n \geq n^{\star}(\delta)$.

Proof. As noted already at the end of Section V condition 37) (which holds for any strongly admissible scaling) implies (24) for all $n=1,2, \ldots$ sufficiently large. On that range Lemma 7.2 yields

$$
1-e^{-\frac{K_{n}^{2}}{P_{n}}} \leq 1-q\left(\theta_{n}\right) \leq \frac{K_{n}^{2}}{P_{n}-K_{n}} .
$$

Multiply (40) by $\frac{P_{n}}{K_{n}^{2}}$ and let $n$ go to infinity in the resulting set of inequalities. Under (37), we get

$$
\lim _{n \rightarrow \infty} \frac{P_{n}}{K_{n}^{2}} \cdot\left(1-e^{-\frac{K_{n}^{2}}{P_{n}}}\right)=1
$$

from the elementary fact $\lim _{t \downarrow 0} \frac{1-e^{-t}}{t}=1$, while

$$
\lim _{n \rightarrow \infty} \frac{P_{n}}{K_{n}^{2}} \cdot \frac{K_{n}^{2}}{P_{n}-K_{n}}=\lim _{n \rightarrow \infty} \frac{P_{n}}{P_{n}-K_{n}}=1
$$

by virtue of (23) (which is implied by (37)). The asymptotic equivalence (38) follows, and the validity of $(36)$ is immediate.

Conversely, under the condition $\lim _{n \rightarrow \infty} q\left(\theta_{n}\right)=1$, we have $0<q\left(\theta_{n}\right)<1$ for all $n$ sufficiently large (by the comment following (7)), and the constraint (24) necessarily holds for all $n=1,2, \ldots$ sufficiently large. On that range, (40) being valid, we conclude to $\lim _{n \rightarrow \infty} e^{-\frac{K_{n}^{2}}{P_{n}}}=1$ under (36). The convergence (37) now follows and the asymptotic equivalence (38) is given by the first part of the proof.

\section{A BASIC UNION BOUND}

Proposition 6.2 will be established with the help of a union bound for the probability appearing at (34) - The approach is similar to the one used for proving the one-law for connectivity in Erdôs-Rényi graphs [2, p. 164] [8, Section 3.4, p. 40] [19. p. 304]:

Fix $n=2,3, \ldots$ and consider positive integers $K$ and $P$ such that $2 K \leq P$. For any non-empty subset $S$ of nodes, i.e., $S \subseteq\{1, \ldots, n\}$, we define the graph $\mathbb{K}(n ; \theta)(S)$ (with vertex set $S)$ as the subgraph of $\mathbb{K}(n ; \theta)$ restricted to the nodes in $S$. We also say that $S$ is isolated in $\mathbb{K}(n ; \theta)$ if there are no edges (in $\mathbb{K}(n ; \theta)$ ) between the nodes in $S$ and the nodes in the complement $S^{c}=\{1, \ldots, n\}-S$. This is characterized by

$$
K_{i}(\theta) \cap K_{j}(\theta)=\emptyset, \quad i \in S, j \in S^{c} .
$$

With each non-empty subset $S$ of nodes, we associate several events of interest: Let $C_{n}(\theta ; S)$ denote the event 
that the subgraph $\mathbb{K}(n ; \theta)(S)$ is itself connected. The event $C_{n}(\theta ; S)$ is completely determined by the $\operatorname{rvs}\left\{K_{i}(\theta), i \in S\right\}$. We also introduce the event $B_{n}(\theta ; S)$ to capture the fact that $S$ is isolated in $\mathbb{K}(n ; \theta)$, i.e.,

$$
B_{n}(\theta ; S):=\left[K_{i}(\theta) \cap K_{j}(\theta)=\emptyset, \quad i \in S, j \in S^{c}\right] .
$$

Finally, we set

$$
A_{n}(\theta ; S):=C_{n}(\theta ; S) \cap B_{n}(\theta ; S) .
$$

The starting point of the discussion is the following basic observation: If $\mathbb{K}(n ; \theta)$ is not connected and yet has no isolated nodes, then there must exist a subset $S$ of nodes with $|S| \geq$ 2 such that $\mathbb{K}(n ; \theta)(S)$ is connected while $S$ is isolated in $\mathbb{K}(n ; \theta)$. This is captured by the inclusion

$$
C_{n}(\theta)^{c} \cap I_{n}(\theta) \subseteq \cup_{S \in \mathcal{N}:|S| \geq 2} A_{n}(\theta ; S)
$$

with $\mathcal{N}$ denoting the collection of all non-empty subsets of $\{1, \ldots, n\}$. A moment of reflection should convince the reader that this union need only be taken over all subsets $S$ of $\{1, \ldots, n\}$ with $2 \leq|S| \leq\left\lfloor\frac{n}{2}\right\rfloor$. Then, a standard union bound argument immediately gives

$$
\begin{aligned}
\mathbb{P}\left[C_{n}(\theta)^{c} \cap I_{n}(\theta)\right] & \leq \sum_{S \in \mathcal{N}: 2 \leq|S| \leq\left\lfloor\frac{n}{2}\right\rfloor} \mathbb{P}\left[A_{n}(\theta ; S)\right] \\
& =\sum_{r=2}^{\left\lfloor\frac{n}{2}\right\rfloor}\left(\sum_{S \in \mathcal{N}_{r}} \mathbb{P}\left[A_{n}(\theta ; S)\right]\right)
\end{aligned}
$$

where $\mathcal{N}_{r}$ denotes the collection of all subsets of $\{1, \ldots, n\}$ with exactly $r$ elements.

For each $r=1, \ldots, n$, we simplify the notation by writing $A_{n, r}(\theta):=A_{n}(\theta ;\{1, \ldots, r\}), B_{n, r}(\theta):=B_{n}(\theta ;\{1, \ldots, r\})$ and $C_{r}(\theta):=C_{n}(\theta ;\{1, \ldots, r\})$. For $r=n$ this notation is consistent with $C_{n}(\theta)$ as defined in Section $\mathrm{V}$. Under the enforced assumptions, exchangeability gives

$$
\mathbb{P}\left[A_{n}(\theta ; S)\right]=\mathbb{P}\left[A_{n, r}(\theta)\right], \quad S \in \mathcal{N}_{r}
$$

and the expression

$$
\sum_{S \in \mathcal{N}_{r}} \mathbb{P}\left[A_{n}(\theta ; S)\right]=\left(\begin{array}{l}
n \\
r
\end{array}\right) \mathbb{P}\left[A_{n, r}(\theta)\right]
$$

follows since $\left|\mathcal{N}_{r}\right|=\left(\begin{array}{l}n \\ r\end{array}\right)$. Substituting into (42) we obtain the key bound

$$
\mathbb{P}\left[C_{n}(\theta)^{c} \cap I_{n}(\theta)\right] \leq \sum_{r=2}^{\left\lfloor\frac{n}{2}\right\rfloor}\left(\begin{array}{l}
n \\
r
\end{array}\right) \mathbb{P}\left[A_{n, r}(\theta)\right] .
$$

Consider a strongly admissible scaling $P, K: \mathbb{N}_{0} \rightarrow \mathbb{N}_{0}$ as in the statement of Proposition 6.2 In the right hand side of (43) we substitute $\theta$ by $\theta_{n}$ by means of this strongly admissible scaling. The proof of Proposition 6.2 will be completed once we show that

$$
\lim _{n \rightarrow \infty} \sum_{r=2}^{\left\lfloor\frac{n}{2}\right\rfloor}\left(\begin{array}{l}
n \\
r
\end{array}\right) \mathbb{P}\left[A_{n, r}\left(\theta_{n}\right)\right]=0
$$

under the appropriate conditions. This approach was used to establish the one-law in Erdős-Rényi graphs [2], [8], [19] where simple bounds can be derived for the probability terms in (44). Our situation is technically more involved and requires more delicate bounding arguments as will become apparent in the forthcoming sections.

\section{Bounding The PRobabilities $\mathbb{P}\left[A_{n, r}(\theta)\right]$ \\ $(r=1, \ldots, n)$}

Again consider positive integers $K$ and $P$ such that $2 K \leq$ $P$. Fix $n=2,3, \ldots$ and pick $r=1, \ldots, n-1$. Since exact expressions are not available for the probability $\mathbb{P}\left[A_{n, r}(\theta)\right]$, we seek instead to provide a bound on this quantity. For reasons that will become apparent shortly, it will be beneficial to focus on the following more general task: Let $\mathcal{F}_{r}$ denotes the $\sigma$-field on $\Omega$ generated by the rvs $K_{1}(\theta), \ldots, K_{r}(\theta)$. We are interested in deriving an upper bound on the probability $\mathbb{P}\left[A_{n, r}(\theta) \cap E\right]$ where $E$ is any $\mathcal{F}_{r}$-measurable event, the original situation corresponding to $E=\Omega$.

In the course of doing so, we shall make use of the rv $U_{r}(\theta)$ given by

$$
U_{r}(\theta):=\left|\cup_{i=1}^{r} K_{i}(\theta)\right| .
$$

The $\mathrm{rv} U_{r}(\theta)$ counts the number of distinct keys issued to the nodes $1, \ldots, r$, so that the bounds

$$
K \leq U_{r}(\theta) \leq \min (r K, P)
$$

always hold.

Thus, pick any $\mathcal{F}_{r}$-measurable event $E$, and note that $C_{r}(\theta)$ is also an $\mathcal{F}_{r}$-measurable event since completely determined by the rvs $K_{1}(\theta), \ldots, K_{r}(\theta)$. It is now plain (41) that

$$
\begin{aligned}
\mathbb{P}\left[A_{n, r}(\theta) \cap E\right] & =\mathbb{P}\left[B_{n, r}(\theta) \cap C_{r}(\theta) \cap E\right] \\
& =\mathbb{E}\left[\mathbf{1}\left[C_{r}(\theta) \cap E\right] \mathbb{P}\left[B_{n, r}(\theta) \mid \mathcal{F}_{r}\right]\right]
\end{aligned}
$$

upon preconditioning on the rvs $K_{1}(\theta), \ldots, K_{r}(\theta)$. Next, with the help of the equivalence

$$
B_{n, r}(\theta)=\left[\left(\cup_{i=1}^{r} K_{i}(\theta)\right) \cap K_{j}(\theta)=\emptyset, j=r+1, \ldots n\right],
$$

we can use (7) (with $S=\cup_{i=1}^{r} K_{i}(\theta)$ ) to get

$$
\begin{aligned}
\mathbb{P} & {\left[B_{n, r}(\theta) \mid \mathcal{F}_{r}\right] } \\
& =\left(\frac{\left(\begin{array}{c}
P-U_{r}(\theta) \\
K
\end{array}\right)}{\left(\begin{array}{l}
P \\
K
\end{array}\right)}\right)^{n-r} \mathbf{1}\left[U_{r}(\theta) \leq P-K\right] \quad \text { a.s. }
\end{aligned}
$$

under the enforced independence assumptions. The conclusion

$\mathbb{P}\left[A_{n, r}(\theta) \cap E\right]=\mathbb{E}\left[\mathbf{1}\left[C_{r}^{\star}(\theta) \cap E\right] \cdot\left(\frac{\left(\begin{array}{c}P-U_{r}(\theta) \\ K\end{array}\right)}{\left(\begin{array}{l}P \\ K\end{array}\right)}\right)^{n-r}\right]$

then follows with

$$
C_{r}^{\star}(\theta):=C_{r}(\theta) \cap\left[U_{r}(\theta) \leq P-K\right] .
$$

Applying (35) (with $L=U_{r}(\theta)$ ) in Lemma 7.1, we finally obtain the inequality

$$
\begin{aligned}
\mathbb{P} & {\left[A_{n, r}(\theta) \cap E\right] } \\
& \leq \mathbb{E}\left[\mathbf{1}\left[C_{r}^{\star}(\theta) \cap E\right] \cdot e^{-(n-r) \frac{K}{P} \cdot U_{r}(\theta)}\right] .
\end{aligned}
$$

This discussion already brings out a number of items that are likely to require some attention: We will need good bounds for the probabilities $\mathbb{P}\left[C_{r}(\theta)\right]$ and $\mathbb{P}\left[C_{r}^{\star}(\theta)\right]$. Also, some of the distributional properties of the $\mathrm{rv} U_{r}(\theta)$ are expected to play a role. The constraints (45) automatically imply $U_{r}(\theta) \leq P-K$ whenever $r K \leq P-K$, i.e., $(r+1) K \leq P$, whence

$$
C_{r}^{\star}(\theta)=C_{r}(\theta), \quad r=1, \ldots, r_{n}(\theta)
$$


where we have set

$$
r_{n}(\theta):=\min \left(r(\theta),\left\lfloor\frac{n}{2}\right\rfloor\right) \quad \text { with } \quad r(\theta):=\left\lfloor\frac{P}{K}\right\rfloor-1 .
$$

This suggests that different arguments will probably be needed for the ranges $1 \leq r \leq r_{n}(\theta)$ and $r_{n}(\theta)<r \leq\left\lfloor\frac{n}{2}\right\rfloor$.

The next result is crucial to showing that for each $r=$ $2 \ldots, n$, the probability of the event $C_{r}(\theta)$ can be provided an upper bound in terms of known quantities. Let $\mathbb{K}_{r}(n ; \theta)$ stand for the subgraph $\mathbb{K}(n ; \theta)(S)$ when $S=\{1, \ldots, r\}$, and let $\mathcal{T}_{r}$ denote the collection of all spanning trees on the vertex set $\{1, \ldots, r\}$.

Lemma 9.1: For each $r=2, \ldots, n$, we have

$$
\mathbb{P}\left[T \subset \mathbb{K}_{r}(n ; \theta)\right]=(1-q(\theta))^{r-1}, \quad T \in \mathcal{T}_{r}
$$

where the notation $T \subset \mathbb{K}_{r}(n ; \theta)$ indicates that the tree $T$ is a subgraph spanning $\mathbb{K}_{r}(n ; \theta)$.

This last expression is analogous to the one found in Erdős-Rényi graphs [2], [8] with $1-q(\theta)$ playing the role of probability of link assignment, and this in spite of the correlations between some link assignments.

Proof. We shall prove the result by induction on $r=2, \ldots, n$. For $r=2$ the conclusion (48) is nothing more than (6) since $\mathcal{T}_{2}$ contains exactly one tree, and this establishes the basis step.

Next, we consider the following induction step: Pick $r=$ $2, \ldots, n-1$ and assume that for each $s=2, \ldots, r$, it is already known that

$$
\mathbb{P}\left[T \subset \mathbb{K}_{s}(n ; \theta)\right]=(1-q(\theta))^{s-1}, \quad T \in \mathcal{T}_{s} .
$$

We now show that 49) also holds for each $s=2, \ldots, r+1$. To that end, pick a tree $T$ in $\mathcal{T}_{r+1}$ and identify its root 3 Let $i$ denote a node that is farthest from the root of $T$ - There might be several such nodes. Also denote by $p$ its unique parent, and let $D(p)$ denote the set of children of $p$. Obviously $D(p)$ is not empty as it contains node $i$; set $|D(p)|=d$. Next we construct a new tree $T^{\star}$ from $T$ by removing from $T$ all the edges from node $p$ to the nodes in $D(p)$. By exchangeability, there is no loss of generality in assuming (as we do from now on) that the tree is rooted at node 1 , that the unique parent $p$ of the farthest node selected has label $r-d+1$, and that its children have been labelled $r-d+2, \ldots, r+1$. With this convention, the tree $T^{\star}$ is defined on the set of nodes $\{1, \ldots, r-d+1\}$.

It is plain that $T \subseteq \mathbb{K}_{r+1}(n, ; \theta)$ occurs if and only if the two sets of conditions

$$
K_{r-d+1}(\theta) \cap K_{\ell}(\theta) \neq \emptyset, \quad \ell=r-d+2, \ldots, r+1
$$

and

$$
T^{\star} \subseteq \mathbb{K}_{r-d+1}(n ; \theta)
$$

both hold. Under the enforced independence assumptions we get

$$
\mathbb{P}\left[\begin{array}{c}
K_{r-d+1}(\theta) \cap K_{\ell}(\theta) \neq \emptyset, \\
\ell=r-d+2, \ldots, r+1
\end{array} \mid \mathcal{F}_{r-d+1}\right]=(1-q(\theta))^{d} .
$$

\footnotetext{
${ }^{3}$ As we are considering undirected graphs, all nodes can act as a root for the (undirected) tree $T$, in which case any one will do for the forthcoming discussion.
}

Thus, upon conditioning with respect to the rvs $K_{1}(\theta), \ldots, K_{r-d+1}(\theta)$ we readily find

$$
\begin{aligned}
\mathbb{P} & {\left[T \subseteq \mathbb{K}_{r+1}(n, ; \theta)\right] } \\
& =(1-q(\theta))^{d} \mathbb{P}\left[T^{\star} \subseteq \mathbb{K}_{r-d+1}(n ; \theta)\right] \\
& =(1-q(\theta))^{d}(1-q(\theta))^{r-d} \\
& =(1-q(\theta))^{r}
\end{aligned}
$$

as we use the induction hypothesis (49) to evaluate the probability of the event $\left[T^{\star} \subseteq \mathbb{K}_{r-d+1}(n ; \theta)\right]$. This establishes the induction step. [8].

The bound below now follows as in Erdős-Rényi graphs [2],

Lemma 9.2: For each $r=2, \ldots, n$, we have

$$
\mathbb{P}\left[C_{r}(\theta)\right] \leq r^{r-2}(1-q(\theta))^{r-1} .
$$

Proof. Fix $r=2, \ldots, n$. If $\mathbb{K}_{r}(n ; \theta)$ is a connected graph, then it must contain a spanning tree on the vertex set $\{1, \ldots . r\}$, and a union bound argument yields

$$
\mathbb{P}\left[C_{r}(\theta)\right] \leq \sum_{T \in \mathcal{T}_{r}} \mathbb{P}[T \subset \mathbb{K}(n ; \theta)(S)] .
$$

By Cayley's formula [3], [15] there are $r^{r-2}$ trees on $r$ vertices, i.e., $\left|\mathcal{T}_{r}\right|=r^{r-2}$, and (50) follows upon making use of (48).

The bound (46) (with $E=\Omega$ ) and the inequality $U_{r}(\theta) \geq K$ together imply

$$
\begin{aligned}
\mathbb{P}\left[A_{n, r}(\theta)\right] & \leq \mathbb{P}\left[C_{r}(\theta)\right] \cdot e^{-(n-r) \frac{K^{2}}{P}} \\
& \leq r^{r-2}(1-q(\theta))^{r-1} \cdot e^{-(n-r) \frac{K^{2}}{P}}
\end{aligned}
$$

as we make use of Lemma 9.2 in the last step. Unfortunately, this bound turns out to be too loose for our purpose. As this can be traced to the crude lower bound used for $U_{r}(\theta)$, we expect that improvements are possible if we take into account the distributional properties of the $\operatorname{rv} U_{r}(\theta)$. This step is taken in the next section.

\section{$\mathrm{X}$. The taIL OF THE RV $U_{r}(\theta)$ AND IMPROVED BoundS}

Consider positive integers $K$ and $P$ such that $K \leq P$. Rough estimates will suffice to get the needed information regarding the distribution of the rv $U_{r}(\theta)$. This is the content of the next result.

Lemma 10.1: For all $r=1,2, \ldots$, the bounds

$$
\mathbb{P}\left[U_{r}(\theta) \leq x\right] \leq\left(\begin{array}{l}
P \\
x
\end{array}\right)\left(\frac{x}{P}\right)^{r K}
$$

holds whenever $x=K, \ldots, \min (r K, P)$.

Proof. For a given $x$ in the prescribed range, we note that $U_{r}(\theta) \leq x$ implies that $\cup_{i=1}^{r} K_{i}(\theta)$ is contained in some set $S$ of size $x$, whence

$$
\left[U_{r}(\theta) \leq x\right] \subseteq \bigcup_{S \in \mathcal{P}_{x}}\left[\cup_{i=1}^{r} K_{i}(\theta) \subseteq S\right]
$$


A standard union bound argument gives

$$
\begin{aligned}
\mathbb{P}\left[U_{r}(\theta) \leq x\right] & \leq \sum_{S \in \mathcal{P}_{x}} \mathbb{P}\left[\cup_{i=1}^{r} K_{i}(\theta) \subseteq S\right] \\
& =\sum_{S \in \mathcal{P}_{x}} \mathbb{P}\left[K_{i}(\theta) \subseteq S, i=1, \ldots, r\right] \\
& =\sum_{S \in \mathcal{P}_{x}} \prod_{i=1}^{r} \mathbb{P}\left[K_{i}(\theta) \subseteq S\right] \\
& =\sum_{S \in \mathcal{P}_{x}}\left(\mathbb{P}\left[K_{1}(\theta) \subseteq S\right]\right)^{r}
\end{aligned}
$$

under the enforced assumptions on the rvs $K_{1}(\theta), \ldots, K_{n}(\theta)$.

Since every subset of size $x$ contains $\left(\begin{array}{l}x \\ K\end{array}\right)$ further subsets of size $K$, we get

$$
\mathbb{P}\left[K_{1}(\theta) \subseteq S\right]=\frac{\left(\begin{array}{l}
x \\
K
\end{array}\right)}{\left(\begin{array}{l}
P \\
K
\end{array}\right)}, \quad S \in \mathcal{P}_{x} .
$$

Substituting this fact into 53) we obtain the inequality

$$
\mathbb{P}\left[U_{r}(\theta) \leq x\right] \leq\left(\begin{array}{c}
P \\
x
\end{array}\right)\left(\frac{\left(\begin{array}{l}
x \\
K
\end{array}\right)}{\left(\begin{array}{l}
P \\
K
\end{array}\right)}\right)^{r K}
$$

from the fact $\left|\mathcal{P}_{x}\right|=\left(\begin{array}{l}P \\ x\end{array}\right)$. Under the enforced conditions it is the case that

$$
\frac{\left(\begin{array}{l}
x \\
K
\end{array}\right)}{\left(\begin{array}{l}
P \\
K
\end{array}\right)}=\prod_{\ell=0}^{K-1}\left(\frac{x-\ell}{P-\ell}\right) \leq\left(\frac{x}{P}\right)^{K}
$$

since $\frac{x-\ell}{P-\ell}$ decreases as $\ell$ increases from $\ell=0$ to $\ell=K-1$, and the inequality (52) follows by using this fact into (54).

The bounds (52) trivially hold with $\mathbb{P}\left[U_{r}(\theta) \leq x\right]=0$ when $x=1, \ldots, K-1$ since we always have $U_{r}(\theta) \geq K$. We shall make repeated use of this fact as follows: For all $n, r=$ $1,2, \ldots$, with $r<n$, we have

$$
\begin{aligned}
\left(\begin{array}{l}
n \\
r
\end{array}\right) \mathbb{P}\left[U_{r}(\theta) \leq x\right] & \leq\left(\begin{array}{c}
n \\
r
\end{array}\right)\left(\begin{array}{c}
P \\
x
\end{array}\right)\left(\frac{x}{P}\right)^{r K} \\
& \leq\left(\begin{array}{c}
\lfloor P / \sigma\rfloor \\
r
\end{array}\right)\left(\begin{array}{c}
P \\
x
\end{array}\right)\left(\frac{x}{P}\right)^{r K}
\end{aligned}
$$

on the range $x=1, \ldots, \min (r K, P)$ whenever $\sigma n \leq P$ for some $\sigma>0$, a condition needed only for the last step and which implies $n \leq\left\lfloor\frac{P}{\sigma}\right\rfloor$ since $n$ is an integer.

We are now in a position to improve on the bound (51).

Lemma 10.2: Consider positive integers $K$ and $P$ such that $K \leq P$. With $n=2,3, \ldots$ and $r=1, \ldots, n$, we have

$$
\begin{aligned}
\mathbb{P}\left[A_{n, r}(\theta)\right] \leq & \mathbb{P}\left[U_{r}(\theta) \leq x\right] e^{-(n-r) \frac{K^{2}}{P}} \\
& +\mathbb{P}\left[C_{r}(\theta)\right] e^{-(n-r) \frac{K}{P}(x+1)}
\end{aligned}
$$

for each positive integer $x$.

Proof. Fix $n=2,3, \ldots$ and pick $r=2, \ldots, n-1$. For each positive integer $x$, consider the decomposition

$$
\begin{aligned}
\mathbb{P}\left[A_{n, r}(\theta)\right]= & \mathbb{P}\left[A_{n, r}(\theta) \cap\left[U_{r}(\theta) \leq x\right]\right] \\
& +\mathbb{P}\left[A_{n, r}(\theta) \cap\left[U_{r}(\theta)>x\right]\right] .
\end{aligned}
$$

Using (46) (with $E=\left[U_{r}(\theta) \leq x\right]$ ) and the bound $U_{r}(\theta) \geq$ $K$, we get

$$
\begin{aligned}
\mathbb{P} & {\left[A_{n, r}(\theta) \cap\left[U_{r}(\theta) \leq x\right]\right] } \\
& \leq \mathbb{P}\left[C_{r}^{\star}(\theta) \cap\left[U_{r}(\theta) \leq x\right]\right] \cdot e^{-(n-r) \frac{K^{2}}{P}} \\
& \leq \mathbb{P}\left[U_{r}(\theta) \leq x\right] \cdot e^{-(n-r) \frac{K^{2}}{P}} .
\end{aligned}
$$

Invoking (46) again (this time with $E=\left[U_{r}(\theta)>x\right]$ ), we find

$$
\begin{aligned}
\mathbb{P} & {\left[A_{n, r}(\theta) \cap\left[U_{r}(\theta) \geq x\right]\right] } \\
& \leq \mathbb{E}\left[\mathbf{1}\left[C_{r}^{\star}(\theta) \cap\left[U_{r}(\theta)>x\right]\right] \cdot e^{-(n-r) \frac{K}{P} \cdot U_{r}(\theta)}\right] \\
& \leq \mathbb{P}\left[C_{r}(\theta)\right] e^{-(n-r) \frac{K}{P}(x+1)}
\end{aligned}
$$

since $U_{r}(\theta) \geq x+1$ on the event $\left[U_{r}(\theta)>x\right]$. We complete the proof by combining (57), 58) and (59).

\section{Outlining the Proof of Proposition 6.2}

It is now clear how to proceed: Consider a strongly admissible scaling $P, K: \mathbb{N}_{0} \rightarrow \mathbb{N}_{0}$ as in the statement of Proposition 6.2 Under (21) we necessarily have $\lim _{n \rightarrow \infty} \frac{P_{n}}{K_{n}}=\infty$ as discussed at the end of Section $\mathrm{V}$, see (23). As a result, $\lim _{n \rightarrow \infty} r_{n}\left(\theta_{n}\right)=\infty$, and for any given integer $R \geq 2$ we have

$$
R<r_{n}\left(\theta_{n}\right), \quad n \geq n^{\star}(R)
$$

for some finite integer $n^{\star}(R)$.

For the time being, pick an integer $R \geq 2$ (to be specified in Section XIII), and on the range $n \geq n^{\star}(R)$ consider the decomposition

$$
\begin{aligned}
\sum_{r=2}^{\left\lfloor\frac{n}{2}\right\rfloor}\left(\begin{array}{l}
n \\
r
\end{array}\right) \mathbb{P}\left[A_{n, r}\left(\theta_{n}\right)\right]= & \sum_{r=2}^{R}\left(\begin{array}{c}
n \\
r
\end{array}\right) \mathbb{P}\left[A_{n, r}\left(\theta_{n}\right)\right] \\
& +\sum_{r=R+1}^{r_{n}(\theta)}\left(\begin{array}{l}
n \\
r
\end{array}\right) \mathbb{P}\left[A_{n, r}\left(\theta_{n}\right)\right] \\
& +\sum_{r=r_{n}\left(\theta_{n}\right)+1}^{\left\lfloor\frac{n}{2}\right\rfloor}\left(\begin{array}{c}
n \\
r
\end{array}\right) \mathbb{P}\left[A_{n, r}\left(\theta_{n}\right)\right] .
\end{aligned}
$$

Let $n$ go to infinity: The desired convergence (44) will be established if we show

$$
\begin{aligned}
& \lim _{n \rightarrow \infty} \sum_{r=2}^{R}\left(\begin{array}{l}
n \\
r
\end{array}\right) \mathbb{P}\left[A_{n, r}\left(\theta_{n}\right)\right]=0, \\
& \lim _{n \rightarrow \infty} \sum_{r=R+1}^{r_{n}\left(\theta_{n}\right)}\left(\begin{array}{l}
n \\
r
\end{array}\right) \mathbb{P}\left[A_{n, r}\left(\theta_{n}\right)\right]=0
\end{aligned}
$$

and

$$
\lim _{n \rightarrow \infty} \sum_{r=r_{n}\left(\theta_{n}\right)+1}^{\left\lfloor\frac{n}{2}\right\rfloor}\left(\begin{array}{l}
n \\
r
\end{array}\right) \mathbb{P}\left[A_{n, r}\left(\theta_{n}\right)\right]=0 .
$$

The next sections are devoted to proving the validity of 62), 63) and 64 by repeated applications of Lemma 10.2 
We address these three cases by making use of the bounds (56) with

$$
\begin{gathered}
x=\left\lfloor(1+\varepsilon) K_{n}\right\rfloor, \quad \varepsilon \in\left(0, \frac{1}{2}\right), \\
x=\left\lfloor\lambda r K_{n}\right\rfloor, \quad \lambda \in(0,1),
\end{gathered}
$$

and

$$
x=\left\lfloor\mu P_{n}\right\rfloor, \quad \mu \in(0,1),
$$

respectively. Throughout, we make repeated use of the standard bounds

$$
\left(\begin{array}{l}
n \\
r
\end{array}\right) \leq\left(\frac{e n}{r}\right)^{r}, \quad \begin{aligned}
& r=1, \ldots, n \\
& n=1,2, \ldots
\end{aligned}
$$

Finally, from convexity we note the inequality

$$
\begin{gathered}
(x+y)^{p} \leq 2^{p-1}\left(x^{p}+y^{p}\right), \quad x, y \geq 0 \\
p \geq 1 .
\end{gathered}
$$

Before getting on the way, we close this section by highlighting key differences between our approach and the one used in the papers [1], [6]. The observation yielding [43, which forms the basis of our discussion, is also used in some form as the starting point in both these references. However, these authors did not take advantage of the fact that the sufficiently tight bound (50) is available for the probability of the event $C_{r}(\theta)$, a consequence of the exact expression (48). Through this bound, we can leverage strong admissibility (via (25)) to get

$$
\left(1-q\left(\theta_{n}\right)\right) \leq(1+\delta) \cdot \frac{K_{n}^{2}}{P_{n}}
$$

for $n$ sufficiently large with any $0<\delta<1$, in which case

$$
\mathbb{P}\left[C_{r}\left(\theta_{n}\right)\right] \leq r^{r-2}\left((1+\delta) \cdot \frac{K_{n}^{2}}{P_{n}}\right)^{r-1}
$$

for each $r=2,3, \ldots, n$. This opens the way to using the properties of the scaling by means of its deviation function defined by (14) - Such a line of arguments cannot be made if the scaling is merely admissible.

The bound (56) arises from the need to efficiently bound the rv $U_{r}\left(\theta_{n}\right)$. Indeed, if it were the case that $U_{r}\left(\theta_{n}\right)=r K_{n}$ for each $r=1, \ldots,\left\lfloor\frac{n}{2}\right\rfloor$, then the conjecture (1)-(2) would readily follow as in Erdős-Rényi graphs by simply making use of the bound (51), e.g., see the arguments in [2], [8], [19]. In addition, the constraint $U_{r}\left(\theta_{n}\right) \leq \min \left(r K_{n}, P_{n}\right)$ already suggests that the cases $r K_{n} \leq P_{n}$ and $P_{n}<r K_{n}$ be considered separately, with a different decomposition (56) on each range - This was also the approach taken in the references [1], [6]. Interestingly enough, a further decomposition of the range $r=1, \ldots,\left\lfloor\frac{P_{n}}{K_{n}}\right\rfloor$ is needed to establish Theorem 4.1. In particular, using the bound (56) with $x=\left\lfloor\lambda r K_{n}\right\rfloor$ for sufficiently small $\lambda$ in $(0,1)$ across the entire range $r=1, \ldots,\left\lfloor\frac{P_{n}}{K_{n}}\right\rfloor$ would not suffice for very small values of $r$ : In that range the obvious bound $U_{r}\left(\theta_{n}\right) \geq K_{n}$ might be tighter than $U_{r}\left(\theta_{n}\right) \geq\left\lfloor\lambda r K_{n}\right\rfloor$, and another form of the bound (56) is needed to obtain the desired results, hence 61.

\section{Establishing 62}

Consider a strongly admissible scaling $P, K: \mathbb{N}_{0} \rightarrow \mathbb{N}_{0}$ whose deviation function $\alpha: \mathbb{N}_{0} \rightarrow \mathbb{R}$ satisfies $\lim _{n \rightarrow \infty} \alpha_{n}=$ $\infty$. According to this scaling, for each $r=2,3, \ldots$ and $n=$ $r+1, r+2, \ldots$, replace $\theta$ by $\theta_{n}$ in Lemma 10.2 with $x=$ $\left\lfloor(1+\varepsilon) K_{n}\right\rfloor$ for some $\varepsilon$ in $\left(0, \frac{1}{2}\right)$. For an arbitrary integer $R \geq 2$, the convergence will follow if we show that

$$
\lim _{n \rightarrow \infty}\left(\begin{array}{l}
n \\
r
\end{array}\right) \mathbb{P}\left[C_{r}\left(\theta_{n}\right)\right] e^{-(n-r) \frac{K_{n}}{P_{n}}\left(\left\lfloor(1+\varepsilon) K_{n}\right\rfloor+1\right)}=0
$$

and

$$
\lim _{n \rightarrow \infty}\left(\begin{array}{l}
n \\
r
\end{array}\right) \mathbb{P}\left[U_{r}\left(\theta_{n}\right) \leq\left\lfloor(1+\varepsilon) K_{n}\right\rfloor\right] e^{-(n-r) \frac{K_{n}^{2}}{P_{n}}}=0
$$

for each $r=2,3, \ldots$. These two convergence statements are established below in Proposition 12.1 and Proposition 12.2. respectively.

Proposition 12.1: Consider a strongly admissible scaling $P, K: \mathbb{N}_{0} \rightarrow \mathbb{N}_{0}$ whose deviation function $\alpha: \mathbb{N}_{0} \rightarrow \mathbb{R}$ satisfies $\lim _{n \rightarrow \infty} \alpha_{n}=\infty$. With $\varepsilon>0$, the convergence (67) holds for each $r=2,3, \ldots$.

Proof. Pick $r=2,3, \ldots$ and $\varepsilon>0$, and consider a strongly admissible scaling $P, K: \mathbb{N}_{0} \rightarrow \mathbb{N}_{0}$. We combine the bounds (50) and 65) to write

$$
\begin{aligned}
& \left(\begin{array}{l}
n \\
r
\end{array}\right) \mathbb{P}\left[C_{r}\left(\theta_{n}\right)\right] e^{-(n-r) \frac{K_{n}}{P_{n}}\left(\left\lfloor(1+\varepsilon) K_{n}\right\rfloor+1\right)} \\
& \leq\left(\frac{e n}{r}\right)^{r} r^{r-2}\left(1-q\left(\theta_{n}\right)\right)^{r-1} e^{-(n-r) \frac{K_{n}}{P_{n}}\left(\left\lfloor(1+\varepsilon) K_{n}\right\rfloor+1\right)} \\
& \leq\left(\frac{e^{r}}{r^{2}}\right) n^{r}\left(1-q\left(\theta_{n}\right)\right)^{r-1} e^{-(n-r) \frac{K_{n}^{2}}{P_{n}}(1+\varepsilon)}
\end{aligned}
$$

for all $n=r+1, r+2, \ldots$. Thus, it follows from Lemma 7.3 (via (38) ) that the convergence 67 will be established if we show that

$$
\lim _{n \rightarrow \infty} n^{r}\left(\frac{K_{n}^{2}}{P_{n}}\right)^{r-1} e^{-(n-r) \frac{K_{n}^{2}}{P_{n}}(1+\varepsilon)}=0 .
$$

This step relies on the strong admissibility of the scaling.

On the range where (69) holds, we find with the help of (14) that

$$
\begin{aligned}
n^{r} & \left(\frac{K_{n}^{2}}{P_{n}}\right)^{r-1} e^{-(n-r) \frac{K_{n}^{2}}{P_{n}}(1+\varepsilon)} \\
& =n^{r}\left(\frac{\log n+\alpha_{n}}{n}\right)^{r-1} \cdot e^{-(n-r) \frac{\log n+\alpha_{n}}{n}(1+\varepsilon)} \\
& =n\left(\log n+\alpha_{n}\right)^{r-1} \cdot e^{-(1+\varepsilon)\left(1-\frac{r}{n}\right) \log n} \cdot e^{-(1+\varepsilon)\left(1-\frac{r}{n}\right) \alpha_{n}} \\
& =n^{1-(1+\varepsilon)\left(1-\frac{r}{n}\right)} \cdot\left(\log n+\alpha_{n}\right)^{r-1} \cdot e^{-(1+\varepsilon)\left(1-\frac{r}{n}\right) \alpha_{n}} \\
& =n^{-\varepsilon+(1+\varepsilon) \frac{r}{n}} \cdot\left(\log n+\alpha_{n}\right)^{r-1} \cdot e^{-(1+\varepsilon)\left(1-\frac{r}{n}\right) \alpha_{n}} \cdot \quad \text { (71) }
\end{aligned}
$$

Under the condition $\lim _{n \rightarrow \infty} \alpha_{n}=\infty$ it is plain that

$$
\lim _{n \rightarrow \infty} n^{-\varepsilon+(1+\varepsilon) \frac{r}{n}}(\log n)^{r-1} e^{-(1+\varepsilon)\left(1-\frac{r}{n}\right) \alpha_{n}}=0
$$

and

$$
\lim _{n \rightarrow \infty} n^{-\varepsilon+(1+\varepsilon) \frac{r}{n}} \alpha_{n}^{r-1} e^{-(1+\varepsilon)\left(1-\frac{r}{n}\right) \alpha_{n}}=0 .
$$

Letting $n$ go to infinity in 71 we readily get 70 by making use of 66. 
Proposition 12.2: Consider a strongly admissible scaling $P, K: \mathbb{N}_{0} \rightarrow \mathbb{N}_{0}$ whose deviation function $\alpha: \mathbb{N}_{0} \rightarrow \mathbb{R}$ satisfies $\lim _{n \rightarrow \infty} \alpha_{n}=\infty$. For every $\varepsilon$ in $\left(0, \frac{1}{2}\right)$, the convergence (68) holds for each $r=2,3, \ldots$.

Proof. Pick $r=2,3, \ldots$ and $\varepsilon$ in $\left(0, \frac{1}{2}\right)$, and consider a strongly admissible scaling $P, K: \mathbb{N}_{0} \rightarrow \mathbb{N}_{0}$. For $n$ sufficiently large, we use (52) with $x=\left\lfloor(1+\varepsilon) K_{n}\right\rfloor$ to obtain

$$
\begin{aligned}
\left(\begin{array}{c}
n \\
r
\end{array}\right) \mathbb{P}\left[U_{r}\left(\theta_{n}\right) \leq\left\lfloor(1+\varepsilon) K_{n}\right\rfloor\right] \\
\leq\left(\begin{array}{c}
n \\
r
\end{array}\right)\left(\begin{array}{c}
P_{n} \\
\left\lfloor K_{n}(1+\varepsilon)\right\rfloor
\end{array}\right)\left(\frac{\left\lfloor K_{n}(1+\varepsilon)\right\rfloor}{P_{n}}\right)^{r K_{n}} \\
\leq n^{r}\left(\frac{e P_{n}}{\left\lfloor K_{n}(1+\varepsilon)\right\rfloor}\right)^{\left\lfloor K_{n}(1+\varepsilon)\right\rfloor}\left(\frac{\left\lfloor K_{n}(1+\varepsilon)\right\rfloor}{P_{n}}\right)^{r K_{n}} \\
\leq n^{r}\left(e^{\frac{\left\lfloor K_{n}(1+\varepsilon)\right\rfloor}{r K_{n}-\left\lfloor K_{n}(1+\varepsilon)\right\rfloor}} \frac{\left\lfloor K_{n}(1+\varepsilon)\right\rfloor}{P_{n}}\right)^{r K_{n}-\left\lfloor K_{n}(1+\varepsilon)\right\rfloor} .
\end{aligned}
$$

The condition $r \geq 2$ implies the inequalities

$$
\frac{\left\lfloor K_{n}(1+\varepsilon)\right\rfloor}{r K_{n}-\left\lfloor K_{n}(1+\varepsilon)\right\rfloor} \leq \frac{1+\varepsilon}{r-(1+\varepsilon)} \leq \frac{1+\varepsilon}{1-\varepsilon}
$$

and

$$
r K_{n}-\left\lfloor K_{n}(1+\varepsilon)\right\rfloor \geq K_{n}(r-(1+\varepsilon))>0 .
$$

Thus, upon setting

$$
\Gamma(\varepsilon):=(1+\varepsilon) e^{\frac{1+\varepsilon}{1-\varepsilon}},
$$

we conclude by strong admissibility (in view of (23) ) that $\Gamma(\varepsilon) \cdot \frac{K_{n}}{P_{n}}<1$ for all $n$ sufficiently large, whence

$$
e^{\frac{\left\lfloor K_{n}(1+\varepsilon)\right\rfloor}{r K_{n}-\left\lfloor K_{n}(1+\varepsilon)\right\rfloor}} \frac{\left\lfloor K_{n}(1+\varepsilon)\right\rfloor}{P_{n}} \leq \Gamma(\varepsilon) \cdot \frac{K_{n}}{P_{n}}<1
$$

on that range.

There we can write

$$
\begin{aligned}
& \left(\begin{array}{l}
n \\
r
\end{array}\right) \mathbb{P}\left[U_{r}\left(\theta_{n}\right) \leq\left\lfloor(1+\varepsilon) K_{n}\right\rfloor\right] \\
& \leq n^{r}\left(\Gamma(\varepsilon) \cdot \frac{K_{n}}{P_{n}}\right)^{r K_{n}-\left\lfloor K_{n}(1+\varepsilon)\right\rfloor} \\
& \leq n^{r}\left(\Gamma(\varepsilon) \cdot \frac{K_{n}}{P_{n}}\right)^{K_{n}(r-1-\varepsilon)} \\
& \leq n^{r}\left(\Gamma(\varepsilon) \cdot \frac{K_{n}}{P_{n}}\right)^{2(r-1-\varepsilon)} \\
& \leq n^{r}\left(\Gamma(\varepsilon) \cdot \frac{K_{n}^{2}}{P_{n}}\right)^{2(r-1-\varepsilon)} \\
& =n^{r}\left(\Gamma(\varepsilon) \cdot \frac{\log n+\alpha_{n}}{n}\right)^{2(r-1-\varepsilon)} \\
& =n^{-r+2+2 \varepsilon}\left(\Gamma(\varepsilon) \cdot\left(\log n+\alpha_{n}\right)\right)^{2(r-1-\varepsilon)}
\end{aligned}
$$

where we obtain (72) upon using the fact $K_{n} \geq 2$. On the other hand we also have

$$
e^{-(n-r) \frac{K_{n}^{2}}{P_{n}}}=e^{-(n-r) \frac{\log n+\alpha_{n}}{n}}=n^{-\left(1-\frac{r}{n}\right)} \cdot e^{-\frac{n-r}{n} \alpha_{n}} .
$$

Therefore, upon multiplying (73) and (74) we see that Proposition 12.1 will follow if we show that

$$
\lim _{n \rightarrow \infty} n^{-r+1+2 \varepsilon+\frac{r}{n}} \cdot\left(\log n+\alpha_{n}\right)^{2(r-1-\varepsilon)} \cdot e^{-\frac{n-r}{n} \alpha_{n}}=0 .
$$

The choice of $\varepsilon$ and $r$ ensures that $r-1-\varepsilon>0$ and $-r+$ $1+2 \varepsilon+\frac{r}{n}<0$ for all $n$ sufficiently large. The condition $\lim _{n \rightarrow \infty} \alpha_{n}=\infty$ now yields

$$
\lim _{n \rightarrow \infty} n^{-r+1+2 \varepsilon+\frac{r}{n}} \cdot(\log n)^{2(r-1-\varepsilon)} \cdot e^{-\frac{n-r}{n} \alpha_{n}}=0
$$

and

$$
\lim _{n \rightarrow \infty} n^{-r+1+2 \varepsilon+\frac{r}{n}} \cdot \alpha_{n}^{2(r-1-\varepsilon)} \cdot e^{-\frac{n-r}{n} \alpha_{n}}=0 .
$$

The desired conclusion (75) follows by making use of 76 and (77) with the help of the inequality (66).

\section{Establishing (63)}

In order to establish (63) we will need two technical facts which are presented in Proposition 13.1 and Proposition 13.2.

Proposition 13.1: Consider a strongly admissible scaling $P, K: \mathbb{N}_{0} \rightarrow \mathbb{N}_{0}$ whose deviation function $\alpha: \mathbb{N}_{0} \rightarrow \mathbb{R}$ satisfies $\lim _{n \rightarrow \infty} \alpha_{n}=\infty$. With $0<\lambda<1$ and integer $R \geq 2$, we then have

$$
\lim _{n \rightarrow \infty} \sum_{r=R+1}^{\left\lfloor\frac{n}{2}\right\rfloor}\left(\begin{array}{l}
n \\
r
\end{array}\right) \mathbb{P}\left[C_{r}\left(\theta_{n}\right)\right] e^{-(n-r) \frac{K_{n}}{P_{n}}\left(\left\lfloor\lambda r K_{n}\right\rfloor+1\right)}=0
$$

whenever $\lambda$ and $R$ are selected so that

$$
2<\lambda(R+1) .
$$

Proposition 13.1 is proved in Section XV Next, set

$$
C(\lambda ; \sigma):=\left(\frac{e^{2}}{\sigma}\right)^{\frac{\lambda}{1-2 \lambda}}, \quad \begin{gathered}
\sigma>0 \\
0<\lambda<\frac{1}{2} .
\end{gathered}
$$

Proposition 13.2: Consider a strongly admissible scaling $P, K: \mathbb{N}_{0} \rightarrow \mathbb{N}_{0}$ whose deviation function $\alpha: \mathbb{N}_{0} \rightarrow \mathbb{R}$ satisfies $\lim _{n \rightarrow \infty} \alpha_{n}=\infty$. If there exists some $\sigma>0$ such that (16) holds for all $n=1,2, \ldots$ sufficiently large, then

$$
\lim _{n \rightarrow \infty} \sum_{r=1}^{r_{n}\left(\theta_{n}\right)}\left(\begin{array}{l}
n \\
r
\end{array}\right) \mathbb{P}\left[U_{r}\left(\theta_{n}\right) \leq\left\lfloor\lambda r K_{n}\right\rfloor\right] e^{-(n-r) \frac{K_{n}^{2}}{P_{n}}}=0
$$

whenever $\lambda$ in $\left(0, \frac{1}{2}\right)$ is selected small enough so that

$$
\max \left(2 \lambda \sigma, \lambda^{1-2 \lambda}, \lambda C(\lambda ; \sigma)\right)<1 .
$$

A proof of Proposition 13.2 can be found in Section XVI Note that for any $\sigma>0, \lim _{\lambda \downarrow 0} \lambda C(\lambda ; \sigma)=0$ and $\lim _{\lambda \downarrow 0} \lambda^{1-2 \lambda}=0$, hence the condition (82) can always be met by suitably selecting $\lambda>0$ small enough.

We now turn to the proof of 63): Keeping in mind Proposition 13.1 and Proposition 13.2 we select $\lambda$ sufficiently small in $\left(0, \frac{1}{2}\right)$ to meet the condition (82) and then pick any integer $R \geq 2$ sufficiently large to ensure (79). Next consider a strongly admissible scaling $P, K: \mathbb{N}_{0} \rightarrow \mathbb{N}_{0}$ whose deviation 
function $\alpha: \mathbb{N}_{0} \rightarrow \mathbb{R}$ satisfies the condition $\lim _{n \rightarrow \infty} \alpha_{n}=\infty$. Then, for each $n \geq n^{\star}(R)$ (with $n^{\star}(R)$ as specified at (60)), replace $\theta$ by $\theta_{n}$ according to this scaling, and for each $r=R+1, \ldots, r_{n}\left(\theta_{n}\right)$, set $x=\left\lfloor\lambda r K_{n}\right\rfloor$ in Lemma 10.2 with $\lambda$ as specified earlier.

With these preliminaries in place, we see from Lemma 10.2 that (63) holds if both limits

$$
\lim _{n \rightarrow \infty} \sum_{r=R+1}^{r_{n}\left(\theta_{n}\right)}\left(\begin{array}{l}
n \\
r
\end{array}\right) \mathbb{P}\left[C_{r}\left(\theta_{n}\right)\right] e^{-(n-r) \frac{K_{n}}{P_{n}}\left(\left\lfloor\lambda r K_{n}\right\rfloor+1\right)}=0
$$

and

$$
\lim _{n \rightarrow \infty} \sum_{r=R+1}^{r_{n}\left(\theta_{n}\right)}\left(\begin{array}{l}
n \\
r
\end{array}\right) \mathbb{P}\left[U_{r}\left(\theta_{n}\right) \leq\left\lfloor\lambda r K_{n}\right\rfloor\right] e^{-(n-r) \frac{K_{n}^{2}}{P_{n}}}=0
$$

hold. However, under (79) and (82), these two convergence statements are immediate from Proposition 13.1 and Proposition 13.2, respectively.

\section{Establishing (64)}

The following two results are needed to establish (64). The first of these results is given next with a proof available in Section XVII

Proposition 14.1: Consider a strongly admissible scaling $P, K: \mathbb{N}_{0} \rightarrow \mathbb{N}_{0}$ whose deviation function $\alpha: \mathbb{N}_{0} \rightarrow \mathbb{R}$ satisfies $\lim _{n \rightarrow \infty} \alpha_{n}=\infty$. If there exists some $\sigma>0$ such that (16) holds for all $n=1,2, \ldots$ sufficiently large, then

$$
\lim _{n \rightarrow \infty} \sum_{r=r_{n}\left(\theta_{n}\right)+1}^{\left\lfloor\frac{n}{2}\right\rfloor}\left(\begin{array}{l}
n \\
r
\end{array}\right) \mathbb{P}\left[U_{r}\left(\theta_{n}\right) \leq\left\lfloor\mu P_{n}\right\rfloor\right] e^{-(n-r) \frac{K_{n}^{2}}{P_{n}}}=0
$$

whenever $\mu$ in $\left(0, \frac{1}{2}\right)$ is selected so that

$$
\max \left(2\left(\sqrt{\mu}\left(\frac{e}{\mu}\right)^{\mu}\right)^{\sigma}, \sqrt{\mu}\left(\frac{e}{\mu}\right)^{\mu}\right)<1 .
$$

We have $\lim _{\mu \downarrow 0}\left(\frac{e}{\mu}\right)^{\mu}=1$, whence $\lim _{\mu \downarrow 0} \sqrt{\mu}\left(\frac{e}{\mu}\right)^{\mu}=0$, and (83) can be made to hold for any $\sigma>0$ by taking $\mu>$ 0 sufficiently small. The second proposition is established in Section XVIII

Proposition 14.2: Consider an admissible scaling $P, K$ : $\mathbb{N}_{0} \rightarrow \mathbb{N}_{0}$ whose deviation function $\alpha: \mathbb{N}_{0} \rightarrow \mathbb{R}$ satisfies $\lim _{n \rightarrow \infty} \alpha_{n}=\infty$. If there exists some $\sigma>0$ such that (16) holds for all $n=1,2, \ldots$ sufficiently large, then

$$
\lim _{n \rightarrow \infty} \sum_{r=r_{n}\left(\theta_{n}\right)+1}^{\left\lfloor\frac{n}{2}\right\rfloor}\left(\begin{array}{l}
n \\
r
\end{array}\right) \mathbb{P}\left[C_{r}\left(\theta_{n}\right)\right] e^{-(n-r) \frac{K_{n}}{P_{n}}\left(\left\lfloor\mu P_{n}\right\rfloor+1\right)}=0
$$

for each $\mu$ in $(0,1)$.

The proof of (64) is now within easy reach: Consider a strongly admissible scaling $P, K: \mathbb{N}_{0} \rightarrow \mathbb{N}_{0}$ whose deviation function $\alpha: \mathbb{N}_{0} \rightarrow \mathbb{R}$ satisfies $\lim _{n \rightarrow \infty} \alpha_{n}=\infty$. On the range where (16) holds, for each $n \geq n^{\star}(R)$ (with $n^{\star}(R)$ as specified at (60) where $R$ and $\lambda$ still satisfy (79) and (82)), replace $\theta$ by $\theta_{n}$ according to this scaling, and set $x=\left\lfloor\mu P_{n}\right\rfloor$ in Lemma 10.2 with $\mu$ as specified by 83). We get 640 as a direct consequence of Proposition 14.1 and Proposition 14.2

\section{A PROOF OF Proposition 13.1}

Let $\lambda$ and $R$ be as in the statement of Proposition 13.1, and pick a positive integer $n$ such that $2(R+1)<n$. Arguments similar to the ones leading to 696 yield

$$
\begin{aligned}
& \left(\begin{array}{c}
n \\
r
\end{array}\right) \mathbb{P}\left[C_{r}\left(\theta_{n}\right)\right] e^{-(n-r) \frac{K_{n}}{P_{n}}\left(\left\lfloor\lambda r K_{n}\right\rfloor+1\right)} \\
& \leq\left(\frac{e^{r}}{r^{2}}\right) n^{r} e^{-\lambda r(n-r) \frac{K_{n}^{2}}{P_{n}}}\left(1-q\left(\theta_{n}\right)\right)^{r-1}
\end{aligned}
$$

for all $r=1, \ldots, n$. Thus, in order to establish (78), we need only show

$$
\lim _{n \rightarrow \infty} \sum_{r=R+1}^{\left\lfloor\frac{n}{2}\right\rfloor} \frac{e^{r}}{r^{2}} n^{r} e^{-\lambda r(n-r) \frac{K_{n}^{2}}{P_{n}}}\left(1-q\left(\theta_{n}\right)\right)^{r-1}=0 .
$$

As in the proof of Proposition 12.2 by the strong admissibility of the scaling (with the help of (39)), it suffices to show

$$
\lim _{n \rightarrow \infty} \sum_{r=R+1}^{\left\lfloor\frac{n}{2}\right\rfloor} \frac{e^{r}}{r^{2}} n^{r} e^{-\lambda r(n-r) \frac{K_{n}^{2}}{P_{n}}}\left((1+\delta) \frac{K_{n}^{2}}{P_{n}}\right)^{r-1}=0
$$

with $0<\delta<1$.

Fix $n=2,3, \ldots$ For each $r=1, \ldots,\left\lfloor\frac{n}{2}\right\rfloor$, we get

$$
\begin{aligned}
& \left(\frac{e^{r}}{r^{2}}\right) n^{r} e^{-\lambda r(n-r) \frac{K_{n}^{2}}{P_{n}}}\left((1+\delta) \frac{K_{n}^{2}}{P_{n}}\right)^{r-1} \\
& =\left(\frac{e^{r}}{r^{2}}\right) n^{r} e^{-\lambda r(n-r) \frac{\log n+\alpha_{n}}{n}}\left((1+\delta) \frac{\log n+\alpha_{n}}{n}\right)^{r-1} \\
& =n\left(\frac{e^{r}}{r^{2}}\right) e^{-\lambda r(n-r) \frac{\log n+\alpha_{n}}{n}}\left((1+\delta)\left(\log n+\alpha_{n}\right)\right)^{r-1} \\
& \leq n e^{r} e^{-\lambda r\left(1-\frac{r}{n}\right)\left(\log n+\alpha_{n}\right)}\left((1+\delta)\left(\log n+\alpha_{n}\right)\right)^{r-1} \\
& \leq n e^{r} e^{-\frac{\lambda}{2} r\left(\log n+\alpha_{n}\right)}\left((1+\delta)\left(\log n+\alpha_{n}\right)\right)^{r-1} \\
& =n\left(e^{1-\frac{\lambda}{2}\left(\log n+\alpha_{n}\right)}\right)^{r}\left((1+\delta)\left(\log n+\alpha_{n}\right)\right)^{r-1}
\end{aligned}
$$

as we note that

$$
1-\frac{r}{n} \geq \frac{1}{2}, \quad r=1, \ldots,\left\lfloor\frac{n}{2}\right\rfloor .
$$

Next, we set

$$
\Gamma_{n}(\lambda):=n e^{1-\frac{\lambda}{2}\left(\log n+\alpha_{n}\right)}
$$

and

$$
a_{n}(\lambda):=e^{1-\frac{\lambda}{2}\left(\log n+\alpha_{n}\right)}(1+\delta)\left(\log n+\alpha_{n}\right) .
$$

With this notation we conclude that

$$
\begin{aligned}
& \sum_{r=R+1}^{\left\lfloor\frac{n}{2}\right\rfloor}\left(\frac{e^{r}}{r^{2}}\right) n^{r} e^{-\lambda r(n-r) \frac{K_{n}^{2}}{P_{n}}}\left((1+\delta) \frac{K_{n}^{2}}{P_{n}}\right)^{r-1} \\
\leq & \Gamma_{n}(\lambda) \sum_{r=R+1}^{\left\lfloor\frac{n}{2}\right\rfloor} a_{n}(\lambda)^{r-1} \\
\leq & \Gamma_{n}(\lambda) \sum_{r=R}^{\infty} a_{n}(\lambda)^{r} .
\end{aligned}
$$


Obviously, $\lim _{n \rightarrow \infty} a_{n}(\lambda)=0$ under the condition $\lim _{n \rightarrow \infty} \alpha_{n}=\infty$, so that $a_{n}(\lambda)<1$ for all $n$ sufficiently large. On that range, the geometric series at 86 ) converges to a finite limit with

$$
\sum_{r=R}^{\infty} a_{n}(\lambda)^{r}=\frac{a_{n}(\lambda)^{R}}{1-a_{n}(\lambda)}
$$

Thus,

$$
\begin{aligned}
& \sum_{r=R+1}^{\left\lfloor\frac{n}{2}\right\rfloor}\left(\frac{e^{r}}{r^{2}}\right) n^{r} e^{-\lambda r(n-r) \frac{K_{n}^{2}}{P_{n}}}\left((1+\delta) \frac{K_{n}^{2}}{P_{n}}\right)^{r-1} \\
& \quad \leq \quad \Gamma_{n}(\lambda) \cdot \frac{a_{n}(\lambda)^{R}}{1-a_{n}(\lambda)} \\
& \quad=\quad C_{n, R}(\delta) \cdot n^{1-\frac{\lambda}{2}(R+1)} \cdot e^{-\frac{\lambda}{2}(R+1) \alpha_{n}} \cdot\left(\log n+\alpha_{n}\right)^{R}
\end{aligned}
$$

with

$$
C_{n, R}(\delta):=\frac{e^{R+1}(1+\delta)^{R}}{1-a_{n}(\lambda)} .
$$

Under (79), the condition $\lim _{n \rightarrow \infty} \alpha_{n}=\infty$ implies

$$
\lim _{n \rightarrow \infty} n^{1-\frac{\lambda}{2}(R+1)} \cdot e^{-\frac{\lambda}{2}(R+1) \alpha_{n}} \cdot(\log n)^{R}=0
$$

and

$$
\lim _{n \rightarrow \infty} n^{1-\frac{\lambda(R+1)}{2}} \cdot e^{-\frac{\lambda(R+1)}{2} \alpha_{n}} \cdot \alpha_{n}^{R}=0 .
$$

The desired conclusion (84) is now immediate with the help of the inequality (66).

\section{A PROOF OF PROPOSITION 13.2}

We begin by providing bounds on the probabilities of interest entering 817. Recall the definitions of the quantities introduced before the statement of Proposition 13.2

Proposition 16.1: Consider positive integers $K, P$ and $n$ such that $2 \leq K \leq P$ and $\sigma n \leq P$ for some $\sigma>0$. For any $\lambda$ in $\left(0, \frac{1}{2}\right)$ small enough to ensure

$$
\max (2 \lambda \sigma, \lambda C(\lambda ; \sigma))<1,
$$

we have

$$
\left(\begin{array}{l}
n \\
r
\end{array}\right) \mathbb{P}\left[U_{r}(\theta) \leq\lfloor\lambda r K\rfloor\right] \leq B(\lambda ; \sigma ; K)^{r}
$$

for all $r=1, \ldots, r_{n}(\theta)$ where we have set

$$
B(\lambda ; \sigma ; K):=\max \left(\lambda^{1-2 \lambda}, \lambda^{1-2 \lambda}\left(\frac{e^{2}}{\sigma}\right)^{\lambda}, \frac{e^{2}}{\sigma K^{K-2}}\right) .
$$

Proof. Pick positive integers $K, P$ and $n$ as in the statement of Proposition 16.1 For each $r=1,2, \ldots, n$, we use (55) with $x=\lfloor\lambda r K\rfloor$ to find

$$
\left(\begin{array}{l}
n \\
r
\end{array}\right) \mathbb{P}\left[U_{r}(\theta) \leq\lfloor\lambda r K\rfloor\right] \leq\left(\begin{array}{c}
\left\lfloor\frac{P}{\sigma}\right\rfloor \\
r
\end{array}\right)\left(\begin{array}{c}
P \\
\lfloor\lambda r K\rfloor
\end{array}\right)\left(\frac{\lfloor\lambda r K\rfloor}{P}\right)^{r K} .
$$

On the range

$$
r=1, \ldots, r_{n}(\theta),
$$

the inequalities

$$
r \leq\left\lfloor\frac{P}{K}\right\rfloor-1<\frac{P}{K}
$$

hold, whence $r<\frac{P}{2}$ since $K \geq 2$. Now if $\lambda$ is selected in $\left(0, \frac{1}{2}\right)$ sufficiently small such that $2 \lambda \sigma<1$, it then follows from 89 that $\lambda r K<\lambda P<\frac{P}{2 \sigma}$ so that

$$
\lfloor\lambda r K\rfloor \leq\left\lfloor\frac{P}{2 \sigma}\right\rfloor \leq \frac{1}{2}\left\lfloor\frac{P}{\sigma}\right\rfloor .
$$

Under these circumstances, we also have

$$
r K-\lfloor 2 \lambda r K\rfloor \geq(1-2 \lambda) r K>0 .
$$

Two possibilities arise:

Case I: $r \leq\lfloor\lambda r K\rfloor-$ Since $r \leq\lfloor\lambda r K\rfloor \leq \frac{1}{2}\left\lfloor\frac{P}{\sigma}\right\rfloor$ by (90), we get

$$
\begin{aligned}
\left(\begin{array}{l}
n \\
r
\end{array}\right) \mathbb{P}\left[U_{r}(\theta) \leq\lfloor\lambda r K\rfloor\right] \\
\leq\left(\begin{array}{c}
\left\lfloor\frac{P}{\sigma}\right\rfloor \\
\lfloor\lambda K\rfloor
\end{array}\right)\left(\begin{array}{c}
P \\
\lfloor\lambda r K\rfloor
\end{array}\right)\left(\frac{\lfloor\lambda r K\rfloor}{P}\right)^{r K} \\
\leq\left(\frac{e\left\lfloor\frac{P}{\sigma}\right\rfloor}{\lfloor\lambda r K\rfloor}\right)^{\lfloor\lambda r K\rfloor}\left(\frac{e P}{\lfloor\lambda r K\rfloor}\right)^{\lfloor\lambda r K\rfloor}\left(\frac{\lfloor\lambda r K\rfloor}{P}\right)^{r K} \\
\leq\left(\frac{e}{\sigma} \frac{P}{\lfloor\lambda r K\rfloor}\right)^{\lfloor\lambda r K\rfloor}\left(\frac{e P}{\lfloor\lambda r K\rfloor}\right)^{\lfloor\lambda r K\rfloor}\left(\frac{\lfloor\lambda r K\rfloor}{P}\right)^{r K} \\
=\left(\frac{e^{2}}{\sigma}\right)^{\lfloor\lambda r K\rfloor}\left(\frac{\lfloor\lambda r K\rfloor}{P}\right)^{r K-2\lfloor\lambda r K\rfloor} \\
=\left(\left(\frac{e^{2}}{\sigma}\right)^{\frac{\lfloor\lambda r K\rfloor}{r K-2\lfloor\lambda r K\rfloor}} \cdot \frac{\lfloor\lambda r K\rfloor}{P}\right)^{r K-2\lfloor\lambda r K\rfloor} \\
\leq\left(\max (1, C(\lambda ; \sigma)) \cdot \frac{\lfloor\lambda r K\rfloor}{P}\right)^{r K-2\lfloor\lambda r K\rfloor}
\end{aligned}
$$

with $C(\lambda ; \sigma)$ given by 80 - In the last step we made use of (91) together with the fact that

$$
\frac{\lfloor\lambda r K\rfloor}{r K-2\lfloor\lambda r K\rfloor} \leq \frac{\lambda r K}{r K-2 \lambda r K}=\frac{\lambda}{1-2 \lambda}
$$

since $\lfloor\lambda r K\rfloor \leq \lambda r K$.

On the range (88), we have $r K \leq P$ from (89) and substituting this fact into 92 yields

$\left(\begin{array}{l}n \\ r\end{array}\right) \mathbb{P}\left[U_{r}(\theta) \leq\lfloor\lambda r K\rfloor\right] \leq(\lambda \max (1, C(\lambda ; \sigma)))^{r K-2\lfloor\lambda r K\rfloor}$.

If $\lambda$ in $\left(0, \frac{1}{2}\right)$ were selected such that $\lambda C(\lambda ; \sigma)<1$, then $\lambda \max (1, C(\lambda ; \sigma))<1$, and we get

$$
\left(\begin{array}{l}
n \\
r
\end{array}\right) \mathbb{P}\left[U_{r}(\theta) \leq\lfloor\lambda r K\rfloor\right] \leq(\lambda \max (1, C(\lambda ; \sigma)))^{(1-2 \lambda) r K}
$$

by recalling (91). With this selection this last upper bound is largest when $K=1$, whence

$$
\begin{aligned}
& \left(\begin{array}{l}
n \\
r
\end{array}\right) \mathbb{P}\left[U_{r}(\theta) \leq\lfloor\lambda r K\rfloor\right] \\
& \leq\left(\max \left(\lambda^{1-2 \lambda}, \lambda^{1-2 \lambda}\left(\frac{e^{2}}{\sigma}\right)^{\lambda}\right)\right)^{r} .
\end{aligned}
$$


Case II: $\lfloor\lambda r K\rfloor \leq r-$ On the range (88), we have $\lfloor\lambda r K\rfloor \leq$ $r \leq \frac{P}{2}$ by virtue of 89 ). This time we find

$$
\begin{aligned}
\left(\begin{array}{c}
n \\
r
\end{array}\right) & \mathbb{P}\left[U_{r}(\theta) \leq\lfloor\lambda r K\rfloor\right] \\
\leq & \left(\begin{array}{c}
\left\lfloor\frac{P}{\sigma}\right\rfloor \\
r
\end{array}\right)\left(\begin{array}{c}
P \\
r
\end{array}\right)\left(\frac{\lfloor\lambda r K\rfloor}{P}\right)^{r K} \\
\leq & \left(\frac{e}{r}\left\lfloor\frac{P}{\sigma}\right\rfloor\right)^{r}\left(\frac{e P}{r}\right)^{r}\left(\frac{\lfloor\lambda r K\rfloor}{P}\right)^{r K} \\
\leq & \left(\frac{e P}{r \sigma}\right)^{r}\left(\frac{e P}{r}\right)^{r}\left(\frac{\lfloor\lambda r K\rfloor}{P}\right)^{r K} .
\end{aligned}
$$

The condition $\lfloor\lambda r K\rfloor \leq r$ now implies

$$
\begin{aligned}
\left(\begin{array}{l}
n \\
r
\end{array}\right) \mathbb{P}\left[U_{r}(\theta) \leq\lfloor\lambda r K\rfloor\right] & \leq\left(\frac{e P}{r \sigma}\right)^{r}\left(\frac{e P}{r}\right)^{r}\left(\frac{r}{P}\right)^{r K} . \\
& =\left(\frac{e^{2}}{\sigma} \cdot\left(\frac{r}{P}\right)^{(K-2)}\right)^{r} \\
& \leq\left(\frac{e^{2}}{\sigma K^{K-2}}\right)^{r}
\end{aligned}
$$

since $r \leq \frac{P}{K}$ upon using 89 . The proof of Proposition 16.1 is completed by combining the inequalities 933 and 944.

We can now turn to the proof of Proposition 13.2. Consider positive integers $K, P$ and $n$ as in the statement of Proposition 16.1 Pick $\lambda$ in $\left(0, \frac{1}{2}\right)$ which satisfies $(82)$ and note that 87 is also valid under this selection. In the usual manner we get

$$
\begin{aligned}
& \sum_{r=1}^{r_{n}(\theta)}\left(\begin{array}{l}
n \\
r
\end{array}\right) \mathbb{P}\left[U_{r}(\theta) \leq\lfloor\lambda r K\rfloor\right] \cdot e^{-(n-r) \frac{K^{2}}{P}} \\
& \leq \sum_{r=1}^{r_{n}(\theta)}\left(\begin{array}{l}
n \\
r
\end{array}\right) \mathbb{P}\left[U_{r}(\theta) \leq\lfloor\lambda r K\rfloor\right] \cdot e^{-\left(n-\left\lfloor\frac{n}{2}\right\rfloor\right) \frac{K^{2}}{P}} \\
& \leq e^{-\frac{n}{2} \frac{K^{2}}{P}} \sum_{r=1}^{r_{n}(\theta)}\left(\begin{array}{l}
n \\
r
\end{array}\right) \mathbb{P}\left[U_{r}(\theta) \leq\lfloor\lambda r K\rfloor\right] \\
& \leq e^{-\frac{n}{2} \frac{K^{2}}{P}} \sum_{r=1}^{r_{n}(\theta)} B(\lambda ; \sigma ; K)^{r}
\end{aligned}
$$

as we invoke Proposition 16.1. If it is the case that $B(\lambda ; \sigma ; K)<1$, the geometric series is summable with

$$
\sum_{r=1}^{r_{n}(\theta)} B(\lambda ; \sigma ; K)^{r} \leq \sum_{r=1}^{\infty} B(\lambda ; \sigma ; K)^{r}=\frac{B(\lambda ; \sigma ; K)}{1-B(\lambda ; \sigma ; K)},
$$

so that

$$
\begin{aligned}
& \sum_{r=1}^{r_{n}(\theta)}\left(\begin{array}{l}
n \\
r
\end{array}\right) \mathbb{P}\left[U_{r}(\theta) \leq\lfloor\lambda r K\rfloor\right] \cdot e^{-(n-r) \frac{K^{2}}{P}} \\
& \leq e^{-\frac{n}{2} \frac{K^{2}}{P}} \frac{B(\lambda ; \sigma ; K)}{1-B(\lambda ; \sigma ; K)} .
\end{aligned}
$$

Now, consider a strongly admissible scaling $P, K: \mathbb{N}_{0} \rightarrow$ $\mathbb{N}_{0}$ whose deviation function $\alpha: \mathbb{N}_{0} \rightarrow \mathbb{R}$ satisfies $\lim _{n \rightarrow \infty} \alpha_{n}=\infty$. On the range where (16) holds, replace $\theta$ by $\theta_{n}$ in the last inequality according to this admissible scaling. From (14) we see that

$$
K_{n}^{2}=\frac{P_{n}}{n}\left(\log n+\alpha_{n}\right) \geq \sigma\left(\log n+\alpha_{n}\right)
$$

so that $\lim _{n \rightarrow \infty} K_{n}=\infty$, whence

$$
\lim _{n \rightarrow \infty}\left(\frac{e^{2}}{\sigma K_{n}^{K_{n}-2}}\right)=0 .
$$

Moreover, any $\lambda$ in the interval $\left(0, \frac{1}{2}\right)$ satisfying 82 also satisfies the condition $\lambda C(\lambda ; \sigma)<1$, so that

$$
\lambda^{1-2 \lambda}\left(\frac{e^{2}}{\sigma}\right)^{\lambda}=(\lambda C(\lambda ; \sigma))^{1-2 \lambda}<1 .
$$

As a result, under 82 we see that

$$
\lim _{n \rightarrow \infty} B\left(\lambda ; \sigma ; K_{n}\right)=\max \left(\lambda^{1-2 \lambda}, \lambda^{1-2 \lambda}\left(\frac{e^{2}}{\sigma}\right)^{\lambda}\right)<1
$$

whence $B\left(\lambda ; \sigma ; K_{n}\right)<1$ for all $n$ sufficiently large. Therefore, on that range (95) is valid under the enforced assumptions with $\theta$ is replaced by $\theta_{n}$, and we obtain

$$
\begin{gathered}
\sum_{r=1}^{r_{n}(\theta)}\left(\begin{array}{l}
n \\
r
\end{array}\right) \mathbb{P}\left[U_{r}\left(\theta_{n}\right) \leq\left\lfloor\lambda r K_{n}\right\rfloor\right] \cdot e^{-(n-r) \frac{K_{n}^{2}}{P_{n}}} \\
\leq e^{-\frac{n}{2} \frac{\log n+\alpha_{n}}{n}} \cdot\left(\frac{B\left(\lambda ; \sigma ; K_{n}\right)}{1-B\left(\lambda ; \sigma ; K_{n}\right)}\right) \\
=n^{-\frac{1}{2}} e^{-\frac{\alpha_{n}}{2}} \cdot\left(\frac{B\left(\lambda ; \sigma ; K_{n}\right)}{1-B\left(\lambda ; \sigma ; K_{n}\right)}\right) .
\end{gathered}
$$

Finally, let $n$ go to infinity in this last expression: The condition $\lim _{n \rightarrow \infty} \alpha_{n}=\infty$ implies $\lim _{n \rightarrow \infty} n^{-\frac{1}{2}} e^{-\frac{\alpha_{n}}{2}}=0$ and this completes the proof.

\section{A PROOF OF Proposition 14.1}

Proposition 14.1 is an easy consequence of the following bound.

Proposition 17.1: Consider positive integers $K$ and $P$ such that $2 \leq K$ and $2 K \leq P$. For each $\mu$ in $\left(0, \frac{1}{2}\right)$, we have

$$
\begin{aligned}
& \sum_{r=r_{n}(\theta)+1}^{\left\lfloor\frac{n}{2}\right\rfloor}\left(\begin{array}{l}
n \\
r
\end{array}\right) \mathbb{P}\left[U_{r}(\theta) \leq\lfloor\mu P\rfloor\right] e^{-(n-r) \frac{K^{2}}{P}} \\
& \leq\left(2 e^{-\frac{K^{2}}{2 P}}\right)^{n}\left(\sqrt{\mu}\left(\frac{e}{\mu}\right)^{\mu}\right)^{P}
\end{aligned}
$$

for all $n=2,3, \ldots$.

Proof. Fix $n=2,3, \ldots$. In establishing (96) we need only consider the case $r_{n}(\theta)<\left\lfloor\frac{n}{2}\right\rfloor$ (for otherwise (96) trivially holds), so that $r_{n}(\theta)=r(\theta)$ and $r_{n}(\theta)+1=\left\lfloor\frac{P}{K}\right\rfloor$. The range $r_{n}(\theta)+1 \leq r \leq\left\lfloor\frac{n}{2}\right\rfloor$ is then equivalent to

$$
\left\lfloor\frac{P}{K}\right\rfloor \leq r \leq\left\lfloor\frac{n}{2}\right\rfloor,
$$

hence

$$
r K \geq\left(\frac{P}{K}-1\right) K \geq \frac{P}{2}
$$


as we make use of the condition $2 K \leq P$ in the last step.

With $\mu$ in the interval $\left(0, \frac{1}{2}\right)$ it follows that

$$
\lfloor\mu P\rfloor \leq \frac{P}{2} \leq \min (r K, P)
$$

and the bound (52) applies with $x=\lfloor\mu P\rfloor$ for all $r=r(\theta)+$ $1, \ldots,\left\lfloor\frac{n}{2}\right\rfloor$.

With this in mind, recall 85 ). We then get

$$
\begin{aligned}
& \sum_{r=r_{n}(\theta)+1}^{\left\lfloor\frac{n}{2}\right\rfloor}\left(\begin{array}{l}
n \\
r
\end{array}\right) \mathbb{P}\left[U_{r}(\theta) \leq\lfloor\mu P\rfloor\right] e^{-(n-r) \frac{K^{2}}{P}} \\
& \leq \sum_{r=r(\theta)+1}^{\left\lfloor\frac{n}{2}\right\rfloor}\left(\begin{array}{c}
n \\
r
\end{array}\right)\left(\begin{array}{c}
P \\
\lfloor\mu P\rfloor
\end{array}\right)\left(\frac{\lfloor\mu P\rfloor}{P}\right)^{r K} e^{-(n-r) \frac{K^{2}}{P}} \\
& \leq e^{-\frac{n}{2} \frac{K^{2}}{P}} \sum_{r=r(\theta)+1}^{\left\lfloor\frac{n}{2}\right\rfloor}\left(\begin{array}{l}
n \\
r
\end{array}\right)\left(\frac{e P}{\lfloor\mu P\rfloor}\right)^{\lfloor\mu P\rfloor}\left(\frac{\lfloor\mu P\rfloor}{P}\right)^{r K} \\
& \leq e^{-\frac{n}{2} \frac{K^{2}}{P}} \sum_{r=r(\theta)+1}^{\left\lfloor\frac{n}{2}\right\rfloor}\left(\begin{array}{l}
n \\
r
\end{array}\right) e^{\lfloor\mu P\rfloor}\left(\frac{\lfloor\mu P\rfloor}{P}\right)^{r K-\lfloor\mu P\rfloor} \\
& \leq e^{-\frac{n}{2} \frac{K^{2}}{P}} \sum_{r=r(\theta)+1}^{\left\lfloor\frac{n}{2}\right\rfloor}\left(\begin{array}{l}
n \\
r
\end{array}\right) e^{\lfloor\mu P\rfloor} \mu^{r K-\lfloor\mu P\rfloor} \\
& \leq e^{-\frac{n}{2} \frac{K^{2}}{P}}\left(\frac{e}{\mu}\right)^{\lfloor\mu P\rfloor}\left(\begin{array}{c}
\left\lfloor\frac{n}{2}\right\rfloor \\
r=r(\theta)+1
\end{array}\left(\begin{array}{l}
n \\
r
\end{array}\right)\right) \mu^{\frac{P}{2}}
\end{aligned}
$$

since $\frac{P}{2} \leq r K$ for all $r=r(\theta)+1, \ldots,\left\lfloor\frac{n}{2}\right\rfloor$ as pointed out earlier. The passage to (97) made use of the fact that $r K-$ $\lfloor\mu P\rfloor \geq 0$. The binomial formula now implies

$$
\sum_{r=r(\theta)+1}^{\left\lfloor\frac{n}{2}\right\rfloor}\left(\begin{array}{l}
n \\
r
\end{array}\right) \leq 2^{n}
$$

so that

$$
\begin{aligned}
& \sum_{r=r_{n}(\theta)+1}^{\left\lfloor\frac{n}{2}\right\rfloor}\left(\begin{array}{l}
n \\
r
\end{array}\right) \mathbb{P}\left[U_{r}(\theta) \leq\lfloor\mu P\rfloor\right] e^{-(n-r) \frac{K^{2}}{P}} \\
& \leq\left(2 e^{-\frac{K^{2}}{2 P}}\right)^{n}\left(\frac{e}{\mu}\right)^{\mu P} \mu^{\frac{P}{2}}
\end{aligned}
$$

and the desired conclusion 967 follows.

Now, if in Proposition 17.1 we assume that $\sigma n \leq P$ for some $\sigma>0$, then the inequality

$$
\left(\sqrt{\mu}\left(\frac{e}{\mu}\right)^{\mu}\right)^{P} \leq\left(\sqrt{\mu}\left(\frac{e}{\mu}\right)^{\mu}\right)^{\sigma n}
$$

follows as soon as

$$
\sqrt{\mu}\left(\frac{e}{\mu}\right)^{\mu}<1
$$

and 96 takes the more compact form

$$
\begin{aligned}
& \sum_{r=r_{n}(\theta)+1}^{\left\lfloor\frac{n}{2}\right\rfloor}\left(\begin{array}{l}
n \\
r
\end{array}\right) \mathbb{P}\left[U_{r}(\theta) \leq\lfloor\mu P\rfloor\right] e^{-(n-r) \frac{K^{2}}{P}} \\
& \leq\left(2 e^{-\frac{K^{2}}{2 P}}\left(\sqrt{\mu}\left(\frac{e}{\mu}\right)^{\mu}\right)^{\sigma}\right)^{n} .
\end{aligned}
$$

To conclude the proof of Proposition 14.1, observe that 99 is implied by selecting $\mu$ in $\left(0, \frac{1}{2}\right)$ according to 83$)$. In that case, consider a strongly admissible scaling $P, K: \mathbb{N}_{0} \rightarrow \mathbb{N}_{0}$. On the range where (16) holds, replace $\theta$ by $\theta_{n}$ in the last inequality according to this scaling. This yields

$$
\begin{aligned}
& \sum_{r=r_{n}\left(\theta_{n}\right)+1}^{\left\lfloor\frac{n}{2}\right\rfloor}\left(\begin{array}{l}
n \\
r
\end{array}\right) \mathbb{P}\left[U_{r}\left(\theta_{n}\right) \leq\left\lfloor\mu P_{n}\right\rfloor\right] e^{-(n-r) \frac{K_{n}^{2}}{P_{n}}} \\
\leq & \left(2 e^{-\frac{K_{n}^{2}}{2 P_{n}}}\left(\sqrt{\mu}\left(\frac{e}{\mu}\right)^{\mu}\right)^{\sigma}\right)^{n} \\
& \leq\left(2\left(\sqrt{\mu}\left(\frac{e}{\mu}\right)^{\mu}\right)^{\sigma}\right)^{n} .
\end{aligned}
$$

Letting $n$ go to infinity in this last inequality, we readily get the desired conclusion from (83).

\section{A PROOF OF PROPOSITION 14.2}

Consider positive integers $K$ and $P$ such that $2 \leq K \leq P$, and pick $\mu$ in the interval $(0,1)$. For each $n=2,3, \ldots$, crude bounding arguments yield

$$
\begin{aligned}
& \sum_{r=r_{n}(\theta)+1}^{\left\lfloor\frac{n}{2}\right\rfloor}\left(\begin{array}{l}
n \\
r
\end{array}\right) \mathbb{P}\left[C_{r}(\theta)\right] \cdot e^{-(n-r) \frac{K}{P}(\lfloor\mu P\rfloor+1)} \\
& \leq \sum_{r=r_{n}(\theta)+1}^{\left\lfloor\frac{n}{2}\right\rfloor}\left(\begin{array}{l}
n \\
r
\end{array}\right) e^{-(n-r) \frac{K}{P}(\mu P)} \\
& \leq\left(\sum_{r=r_{n}(\theta)+1}^{\left\lfloor\frac{n}{2}\right\rfloor}\left(\begin{array}{l}
n \\
r
\end{array}\right)\right) e^{-\frac{n}{2} K \mu} \\
& \leq 2^{n} e^{-\frac{n}{2} K \mu}
\end{aligned}
$$

where we have used (85) and 987.

To complete the proof of Proposition 14.2 consider an admissible scaling $P, K: \mathbb{N}_{0} \rightarrow \mathbb{N}_{0}$ whose deviation function $\alpha: \mathbb{N}_{0} \rightarrow \mathbb{R}$ satisfies $\lim _{n \rightarrow \infty} \alpha_{n}=\infty$. Replace $\theta$ by $\theta_{n}$ in (100) according to this admissible scaling so that

$\sum_{r=r_{n}\left(\theta_{n}\right)+1}^{\left\lfloor\frac{n}{2}\right\rfloor}\left(\begin{array}{l}n \\ r\end{array}\right) \mathbb{P}\left[C_{r}\left(\theta_{n}\right)\right] e^{-(n-r) \frac{K_{n}}{P_{n}}\left\lfloor\mu P_{n}\right\rfloor} \leq\left(2 e^{-\frac{\mu K_{n}}{2}}\right)^{n}$.

Let $n$ go to infinity in this last inequality: The condition (16) implies

$$
K_{n}^{2}=\frac{\log n+\alpha_{n}}{n} \cdot P_{n} \geq \sigma\left(\log n+\alpha_{n}\right)
$$

for $n=1,2, \ldots$ sufficiently large, whence $\lim _{n \rightarrow \infty} K_{n}=\infty$ under the assumed condition $\lim _{n \rightarrow \infty} \alpha_{n}=\infty$. Consequently,

$$
\lim _{n \rightarrow \infty}\left(2 e^{-\frac{\mu K_{n}}{2}}\right)=0
$$

and the desired conclusion follows.

\section{ACKNOWLEDGMENT}

The authors thank the anonymous reviewers for their careful reading of the original manuscript; their comments helped improve the final version of this paper. 


\section{REFERENCES}

[1] S.R. Blackburn and S. Gerke, "Connectivity of the uniform random intersection graph," Discrete Mathematics 309 (2009), pp. 5130-5140.

[2] B. Bollobás, Random Graphs, Second Edition, Cambridge Studies in Advanced Mathematics, Cambridge University Press, Cambridge (UK), 2001.

[3] A. Cayley, "A theorem on trees," Quarterly Journal of Mathematics 23 (1889), pp. 376-378.

[4] H. Chen, A. Perrig and D. Song, "Random key predistribution schemes for sensor networks," in Proceedings of the 2003 IEEE Symposium on Security and Privacy (S\&P 2003), Oakland (CA), May 2003, pp. 197213.

[5] F. Chung and L. Lu, "The diameter of sparse random graphs," Advances in Applied Mathematics 26, 2001, pp. 257-279.

[6] R. Di Pietro, L.V. Mancini, A. Mei, A. Panconesi and J. Radhakrishnan, "Sensor networks that are provably secure," in Proceedings of SecureComm 2006, the 2nd IEEE/CreateNet International Conference on Security and Privacy in Communication Networks, Baltimore (MD), August 2006.

[7] R. Di Pietro, L.V. Mancini, A. Mei, A. Panconesi and J. Radhakrishnan, "Redoubtable sensor networks," ACM Transactions on Information Systems Security TISSEC 11 (2008), pp. 1-22.

[8] M. Draief and L. Massoulié, Epidemics and Rumours in Complex Networks, London Mathematical Society Lecture Notes Series 369, Cambridge University Press, Cambridge (UK), 2010.

[9] L. Eschenauer and V.D. Gligor, "A key-management scheme for distributed sensor networks," in Proceedings of the 9th ACM Conference on Computer and Communications Security (CCS 2002), Washington (DC), November 2002, pp. 41-47.

[10] E. Godehardt and J. Jaworski "Two models of random intersection graphs for classification," in Studies in Classification, Data Analysis and Knowledge Organization 22, Eds. O. Optiz and M. Schwaiger, Springer, Berlin (2003), pp. 67-82.

[11] E. Godehardt, J. Jaworski and K. Rybarczyk, "Random intersection graphs and classification," in Studies in Classification, Data Analysis and Knowledge Organization 33, Eds. H.J. Lens and R. Decker, Springer, Berlin (2007), pp. 67-74

[12] S. Janson, T. Łuczak and A. Ruciński, Random Graphs, WileyInterscience Series in Discrete Mathematics and Optimization, John Wiley \& Sons, 2000.

[13] M.K. Karoński, E.R. Scheinerman and K.B. Singer-Cohen, "On random intersection graphs: The subgraph problem," Combinatorics, Probability and Computing 8 (1999), pp. 131-159.

[14] P. Marbach, "A lower-bound on the number of rankings required in recommender systems using collaborative filtering," Proceedings of the 42nd Annual Conference on Information Sciences and Systems (CISS 2008), Princeton University, Princeton (NJ), March 2008.

[15] G.E. Martin, Counting: The Art of Enumerative Combinatorics, Springer Verlag New York, 2001.

[16] A. Perrig, J. Stankovic and D. Wagner, "Security in wireless sensor networks," Communications of the ACM 47 (2004), pp. 53-57.

[17] K. Rybarczyk, "Diameter of the uniform random intersection graph with a note on the connectivity and the phase transition," Discrete Mathematics 311 (2011), pp. 1998-2019.

[18] K.B. Singer, Random Intersection Graphs, Ph.D. Thesis, Department of Mathematical Sciences, The Johns Hopkins University, Baltimore (MD), 1995.

[19] J. Spencer, "Nine Lectures on Random Graphs," in Ecole d'Eté de Probabilités de Saint Flour XXI - 1991, Editor P.L. Hennequin, Springer Lecture Notes in Mathematics 1541, Springer-Verlag Berlin Heidelberg 1993. pp. 293-347.

[20] D.-M. Sun and B. He, "Review of key management mechanisms in wireless sensor networks," Acta Automatica Sinica 12 (2006), pp. 900906.

[21] Y. Wang, G. Attebury and B. Ramamurthy, "A survey of security issues in wireless sensor networks," IEEE Communications Surveys \& Tutorials 8 (2006), pp. 2-23.

[22] O. Yağan and A.M. Makowski, "On the random graph induced by a random key predistribution scheme under full visibility," In Proceedings of the IEEE International Symposium on Information Theory (ISIT 2008), Toronto (ON), June 2008.

[23] O. Yağan and A.M. Makowski, Zero-One Laws for Connectivity in Random Key Graphs, ISR TechnIcal Report 2009-1, Institute for Systems Research, University of Maryland, College Park (MD), January 2009. Available online at http://hdl.handle.net/1903/8716
[24] O. Yağan and A.M. Makowski, "Connectivity results for random key graphs," In Proceedings of the IEEE International Symposium on Information Theory (ISIT 2009), Seoul (S. Korea), June 2009.

[25] O. Yağan and A. M. Makowski, "On the existence of triangles in random key graphs," in Proceedings of the 47th Annual Allerton Conference on Communication, Control, and Computing, Monticello (IL), September 2009,

[26] O. Yağan and A. M. Makowski, "Connectivity in random graphs induced by a key predistribution scheme: Small key pools," in Proceedings of the 44th Annual Conference on Information Sciences and Systems (CISS 2010), March 2010

[27] O. Yağan and A.M. Makowski, "A zero-one law for the existence of triangles in random key graphs." Available online at http://hdl.handle.net/1903/9403 (Original version) and at http://hdl.handle.net/1903/1215 (Revised version).

[28] O. Yağan, Random Graph Modeling of Key Distribution Schemes in Wireless Sensor Networks, Ph.D. Thesis, Department of Electrical and Computer Engineering, University of Maryland, College Park (MD), June 2011.

Osman Yağan (S'07) received the B.S. degree in Electrical and Electronics Engineering from the Middle East Technical University, Ankara (Turkey) in 2007, and the Ph.D degree in Electrical and Computer Engineering from the University of Maryland, College Park, MD in 2011.

He was a visiting Postdoctoral Scholar at Arizona State University during Fall 2011. Since December 2011, he has been a Postdoctoral Fellow with CyLab at Carnegie Mellon University. His research interests include security in wireless networks, percolation theory, random graphs and their applications.

Armand M. Makowski (M'83-SM'94-F'06) received the Licence en Sciences Mathématiques from the Université Libre de Bruxelles in 1975, the M.S. degree in Engineering-Systems Science from U.C.L.A. in 1976 and the Ph.D. degree in Applied Mathematics from the University of Kentucky in 1981. In August 1981, he joined the faculty of the Electrical Engineering Department at the University of Maryland College Park, where he is Professor of Electrical and Computer Engineering. He has held a joint appointment with the Institute for Systems Research since its establishment in 1985.

Armand Makowski was a C.R.B. Fellow of the Belgian-American Educational Foundation (BAEF) for the academic year 1975-76; he is also a 1984 recipient of the NSF Presidential Young Investigator Award and became an IEEE Fellow in 2006.

His research interests lie in applying advanced methods from the theory of stochastic processes to the modeling, design and performance evaluation of engineering systems, with particular emphasis on communication systems and networks. 